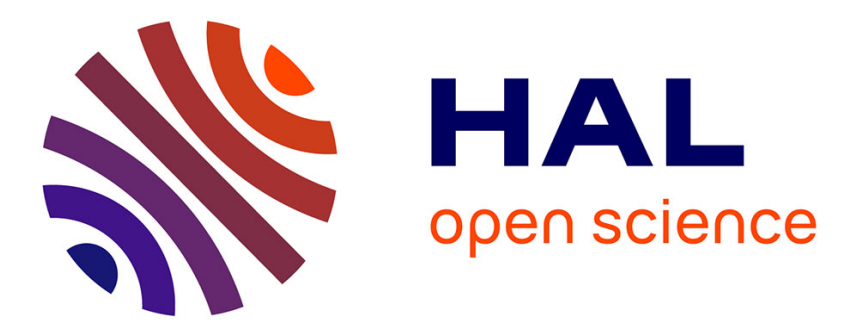

\title{
Comportements alimentaires en Gaule du Nord : étude isotopique du site de l'Ilot de La Boucherie (IIIe-Ve siècles apr. J.-C.) à Amiens
}

Leïa Mion, Estelle Herrscher, Joël J Blondiaux, Guy André, Eric Binet

\section{- To cite this version:}

Leïa Mion, Estelle Herrscher, Joël J Blondiaux, Guy André, Eric Binet. Comportements alimentaires en Gaule du Nord: étude isotopique du site de l'Ilot de La Boucherie (IIIe-Ve siècles apr. J.-C.) à Amiens. Bulletins et Mémoires de la Société d'anthropologie de Paris, 2016, 28 (3-4), pp.155-175. 10.1007/s13219-016-0164-7 . hal-01410444

\author{
HAL Id: hal-01410444 \\ https://hal.science/hal-01410444
}

Submitted on 24 Apr 2019

HAL is a multi-disciplinary open access archive for the deposit and dissemination of scientific research documents, whether they are published or not. The documents may come from teaching and research institutions in France or abroad, or from public or private research centers.
L'archive ouverte pluridisciplinaire $\mathbf{H A L}$, est destinée au dépôt et à la diffusion de documents scientifiques de niveau recherche, publiés ou non, émanant des établissements d'enseignement et de recherche français ou étrangers, des laboratoires publics ou privés. 


\title{
Comportements alimentaires en Gaule du Nord : Etude isotopique du site de l'Ilot de la Boucherie $\left(\mathrm{III}^{\mathrm{e}}-\mathrm{V}^{\mathrm{e}}\right.$ siècles ap $\left.\mathrm{JC}\right)$ à Amiens
}

\section{Dietary patterns in North of Gaule : Isotope evidence from the site of Ilot de la Boucherie (3rd-4 ${ }^{\text {th }}$ centuries AD) in Amiens}

\author{
LEÏA MION ${ }^{1}$, ESTELLE HERRSCHER ${ }^{1}$, JOËL BLONDIAUX ${ }^{2}$, ERIC BINET ${ }^{3}$, \\ GUY ANDRE ${ }^{1}$
}

1 Aix Marseille Univ, CNRS, Minist Culture, LAMPEA, Aix-en-Provence, France

2 Centre d'Etudes Paléopathologiques du Nord, 36 rue Jules Ferry, 59127 WalincourtSelvigny

3 Service Archéologique d'Amiens Métropole, HALMA- IPEL, UMR 8164

Contact : mion.leia@gmail.com

Mots-clés : isotopes stables, collagène, apatite, régime alimentaire, Gaule du Nord, Antiquité Tardive

Key Words : stable isotopes, collagen, bioapatite, diet, Northern Gaul, Late Antiquity

\section{$\underline{\text { RESUME }}$}

L'analyse des comportements alimentaires constitue un indicateur permettant d'approcher les transformations des modes de vie ou encore les processus d'acculturation des populations au cours du temps. C'est notamment dans le contexte particulier de la Gaule du Nord, entre le $\mathrm{III}^{\mathrm{e}}$ siècle et le début du $\mathrm{V}^{\mathrm{e}}$ siècle apr. JC, qu'une étude des pratiques alimentaires a été entreprise afin de cerner l'impact de la romanisation sur ces habitudes. Cette étude propose de contribuer à cette question par l'analyse des isotopes stables du carbone et de l'azote $\left(\delta^{13} \mathrm{C}\right.$ et $\left.\delta^{15} \mathrm{~N}\right)$ contenus dans les phases organique et minérale des dents et des os de sujets adultes provenant du site de l'îlot de la Boucherie à Amiens (Somme). Les objectifs sont de définir le régime alimentaire et de décrire son évolution au cours de la vie des individus. Les sujets échantillonnés $(\mathrm{n}=33)$ forment un groupe aux habitudes alimentaires peu disparates qui ne varient pas entre l'enfance et l'âge adulte. La comparaison des valeurs de $\delta^{13} \mathrm{C}$ entre les sujets humains et les animaux domestiques $(n=28)$ met en 
évidence un enrichissement important en ${ }^{13} \mathrm{C}$ dans les tissus humains, lié soit à la consommation d'une ressource de type C4 comme le millet, soit à celle de ressources marines de faible niveau trophique (fruits de mer). Les valeurs de $\delta^{15} \mathrm{~N}$ des humains relativement basses laissent supposer une alimentation contenant peu de protéines animales. Le régime alimentaire décrit pour cette population se distingue des résultats publiés pour le reste de l'Empire romain.

\section{ABSTRACT}

Analyses of dietary patterns can provide indication on changing lifestyles and acculturation processes in populations overtime. In this study, focusing on the specific context of northern Gaul between the third century and the early fifth century AD, we attempted we attempted to identify the impact of Roman culture on dietary patterns. The aim was to contribute to this research question by analysing the stable carbon and nitrogen isotopes $\left(\delta^{13} \mathrm{C}\right.$ and $\delta^{15} \mathrm{~N}$ ) contained in the organic and inorganic phases of the teeth and bones of adult subjects from the Îlot de la Boucherie site in Amiens (Somme, Northern France) defining the contemporary diet and how it evolved over the lifetime of individuals. The subjects sampled ( $\mathrm{n}=33$ ) form a homogenous group regarding their diet, with no significant variation between childhood and adulthood. A comparison of $\delta^{13} \mathrm{C}$ values in humans and domestic animals $(n=28)$ showed that the human tissues were significantly richer in $13 \mathrm{C}$, reflecting the consumption of either a type $\mathrm{C} 4$ resource such as millet or sea food resources at a low level in the food chain, such as shellfish. The relatively low $\delta^{15} \mathrm{~N}$ values in the human sample suggest a diet containing little animal protein. The diet thus described in this population differs from the results published for the rest of the Roman Empire. 
$\mathrm{Au}$ cours des dix dernières années, les études biochimiques portant sur des sites européens datés de la fin de la période antique ( $\mathrm{II}^{\mathrm{e}}-\mathrm{V}^{\mathrm{e}}$ ap JC) se sont multipliées. Les rapports isotopiques notamment du carbone et de l'azote dosés dans les tissus squelettiques étant corrélés à ceux des ressources consommées au cours de la vie [1], ces études ont permis de mettre en évidence des traits communs à l'ensemble de l'Empire en termes d'alimentation.

Ces études questionnent l'uniformisation culturelle supposée que la conquête romaine implique dans l'Europe de l'Ouest. Aucun site gaulois n'a jusqu'à présent fait l'objet d'une telle étude. Ainsi, cette étude se propose de combler cette lacune en analysant des données concernant le site de l'Îlot de la Boucherie à Amiens en activité du milieu du III $^{\mathrm{e}}$ au début du $\mathrm{V}^{\mathrm{e}}$ siècle ap JC (FIG.1) et se trouvant à proximité des frontières nord de l'Empire. La population particulière inhumée dans ce site, aux caractéristiques morphologiques proches des populations du Sud de l'Allemagne [2] permet d'aborder la question de la spécificité du régime des habitants d'origine germanique en Gaule du Nord et la possible modification de ce régime au cours de la vie des individus.

\section{L'ALIMENTATION DURANT L'ANTIQUITE}

Pour l'Antiquité tardive, les sources historiques indiquent un régime alimentaire fondé sur les céréales [3] généralement du blé ou de l'avoine. La consommation de millet est, elle aussi, attestée par les sources historiques mais se limite aux temps de disette ou aux sujets de bas-statut social [3,4]. L'apport en protéines semble être fonction du statut social : les individus les moins favorisés consomment principalement des légumineuses, dites «viande du pauvre », ou des produits laitiers, tandis que les individus plus favorisés ont plus facilement accès à la viande et au poisson [5]. Les études archéobotaniques, concernant plus spécifiquement la Gaule du Nord pendant la période antique, confirment une agriculture essentiellement fondée sur les céréales avec une prédominance des blés et de l'orge [6]. L'emphase sur les blés nus de type froment laisse penser à une consommation préférentielle de pains à pâte levée. Le millet commun est également présent dans cette partie de la Gaule tout comme l'avoine bien qu'il soit difficile de déterminer si ces céréales sont à destination des humains ou de la faune d'élevage [6,7]. Les légumineuses jouent également un rôle important dans la production agricole du nord de la Gaule et donc par extension, dans l'alimentation des populations de cette région. Ce sont principalement les lentilles, les pois et les féveroles qui sont consommés [6,8,9]. Au Bas Empire, la production de légumineuses tend à augmenter démontrant de manière indirecte l'importance de ces ressources dans le régime alimentaire [6]. En ce qui concerne l'alimentation carnée, les études archéozoologiques 
mettent en avant une présence plus importante du bœuf dans les assemblages relativement au mouton ou au cochon $[6,10]$. Ces études documentent également la présence de poissons marins (grondins, turbot, carrelets) et d'eau douce (flets) à Amiens, ville située à $50 \mathrm{~km}$ de la mer et dans une zone marécageuse sur les rives de la Somme [9]. L'archéologie amiénoise documente également la consommation de produits d'importation tels que le vin, l'huile d'olive et les salaisons de poissons marins [10,11].

Ces informations dressent un tableau global de la production agricole en Gaule du Nord, mais ne permettent pas d'inférer de manière directe sur la contribution de ces différentes ressources au régime des populations de cette région à l'inverse des isotopes stables. Si notre étude est la première concernant cette région, la méthode des isotopes stables a déjà montré tout son intérêt pour des sites de cette période dans le reste de l'Europe (et même au-delà). Les résultats obtenus pour plusieurs zones de l'Empire permettent de mettre en évidence les points communs et les particularités du régime de chaque population composant l'Empire.

La zone géographique la mieux connue par l'outil isotopique est l'Angleterre [12-22]. Les études ont mis en évidence que l'essentiel des ressources consommées sont issues d'un environnement terrestre à la végétation de type $\mathrm{C} 3$ comme c'était le cas avant la conquête romaine. L'influence impériale se fait sentir par la consommation significativement plus importante qu'aux époques précédentes, de ressources issues d'un environnement marin [12,17-19]. La consommation de ressources dulcicoles semble être anecdotique comme à Queenford [13,14].

Les études portant sur le reste du territoire impérial (Espagne [23,24], Tunisie [4], Italie [5,25-29], Bavière [30], Pologne [31], Turquie [32] et Croatie [33]) ont pour la plupart mis en évidence un régime alimentaire similaire fondé principalement sur les ressources terrestres et C3 avec la consommation non négligeable de ressources marines. La part de ressources marines dans l'alimentation semble, comme en Angleterre, liée à l'influence culturelle romaine (eg [33]). La consommation de ressources issues d'un environnement C4 est décelée dans quatre études [4,29,31,32]. Pour les individus étudiés en Tunisie et en Turquie $[4,32]$, cela s'explique essentiellement par la présence de ressources végétales C4 à l'état naturel dans l'environnement direct et leur consommation par la faune. Quant aux populations de Pologne et de Rome [29,31], elles ont, d'après les auteurs, une alimentation comprenant une part importante de céréale $\mathrm{C} 4$ : le millet. Cette consommation serait à mettre en lien avec le faible statut socio-économique des individus [29]. Ce statut influencerait également la quantité de ressources marines dans l'alimentation [5,22,25-27] de même que la 
quantité de protéines d'origine animale [12,27], les «pauvres » consommant moins de ressources marines ou de produits animaux.

\section{MATERIEL}

Le site de l'îlot de la Boucherie a fait l'objet de fouilles archéologiques de sauvetage par l'INRAP en 2006-2007 sous la direction d'Éric Binet. Dans les niveaux d'occupation de la nécropole, 255 sépultures ont été retrouvées. L'étude du mobilier archéologique et les datations radiocarbones permettent de déterminer que l'occupation de la nécropole est comprise entre le milieu du $\mathrm{III}^{\mathrm{e}}$ siècle et le début du $\mathrm{V}^{\mathrm{e}}$ siècle apr. JC. La nécropole a livré les squelettes de 251 sujets adultes et 121 immatures.

La plupart des sépultures ne contenaient pas ou peu de mobilier funéraire ce qui a été rapproché de l'apparition du christianisme à Amiens mais également de la pauvreté relative des sujets inhumés [34,35]. La population constitutive de la nécropole de l'Ilot de la Boucherie semble être exogène et habituée aux travaux physiques lourds. Elle semble être un exemple de la politique de contrôle des territoires de l'Empire par le déplacement forcé de populations germaniques en Gaule Belgique et avoir été utilisée dans le cadre de la construction du castrum qui intervient à peu près à l'époque d'utilisation de la nécropole [2].

Cette étude concerne 33 individus (Tableaux 1,2) sélectionnés aléatoirement dans les différents groupes définis par le secteur d'inhumation, le sexe et l'orientation des sépultures. Les études anthropologiques [2] ont été réalisées par J. Blondiaux et permettent de disposer pour chaque individu de son âge au décès (mesuré par compte des anneaux du cément) et de son sexe [36,37]. Pour chacun des individus, un fragment d'os compact provenant d'une phalange, d'un métacarpe ou d'une côte (dosage du collagène et de l'apatite) a été prélevé ainsi qu'une prémolaire ( $\mathrm{P} 1$ ou $\mathrm{P} 2$ ) dont la période de formation se situe entre 6-14 ans pour la racine (dosage du collagène au niveau de la dentine) et 2-6 ans pour la couronne (dosage de l'apatite au niveau de l'émail) [38,39].

Afin de caractériser isotopiquement l'environnement local, ont également été échantillonnés 10 individus de Sus scrofa, 11 individus de Bos taurus, 3 Ovis aries, 2 Equus caballus, 1 Gallus sp. et 1 Canis familiaris. Ils proviennent de sites d'habitat d'Amiens (21 rue Jules Lardière, Ange d'Or et Saint Rémi) et de Duisans (Tableau 3).

\section{METHODES}

Principes de l'analyse biochimique 
Dans les études visant la restitution de l'alimentation des populations archéologiques, les analyses isotopiques concernent majoritairement deux éléments chimiques : le carbone et l'azote. Notés respectivement $\delta^{13} \mathrm{C}$ et $\delta^{15} \mathrm{~N}$, les rapports isotopiques correspondent à la mesure du rapport entre l'isotope lourd et rare $\left({ }^{13} \mathrm{C},{ }^{15} \mathrm{~N}\right)$ et l'isotope léger et courant $\left({ }^{12} \mathrm{C},{ }^{14} \mathrm{~N}\right)$. Ils peuvent se mesurer sur les deux fractions des tissus osseux et dentaires : le collagène (fraction organique) et l'apatite (fraction minérale) pour le carbone et uniquement sur le collagène pour l'azote. Les informations relatives à l'alimentation enregistrées dans ces deux fractions ne sont pas équivalentes. Les molécules de collagène comprennent des atomes de carbone et d'azote provenant essentiellement de la portion protéinique de l'alimentation [40]. Ainsi, les valeurs de $\delta^{15} \mathrm{~N}$ et $\delta^{13} \mathrm{C}_{\mathrm{co}}$ du collagène des consommateurs renvoient directement aux signatures des protéines alimentaires avec un enrichissement de l'ordre de $0-1 \%$ en ${ }^{13} \mathrm{C}$ et de $3-5 \%$ en ${ }^{15} \mathrm{~N}$ correspondant au fractionnement isotopique entre deux niveaux trophiques consécutifs $[41,42]$. En revanche, les molécules d'apatite comprennent des atomes de carbone issus de l'ensemble de l'alimentation [43,44]. Ainsi, les valeurs de $\delta^{13} C_{a p}$ renvoient aux compositions isotopiques des parties protéinique, glucidique et lipidique de l'alimentation. La comparaison des valeurs de $\delta^{13} \mathrm{C}$ entre le collagène et l'apatite permet donc d'obtenir une information complète sur les habitudes alimentaires et de quantifier notamment la place des protéines dans le régime alimentaire.

Que ce soit au niveau du collagène ou de l'apatite, les compositions isotopiques du carbone permettent de caractériser le type d'environnement dont sont issus les aliments. En effet, les valeurs de $\delta^{13} \mathrm{C}$ des végétaux dépendent :

- du processus de photosynthèse utilisé (C3, C4, CAM) ;

- de la source du carbone, gazeuse ou dissoute, impliquée dans la photosynthèse ;

- des conditions du milieu dans lesquelles s'opèrent la photosynthèse.

Ceci permet de différencier :

- les végétaux « $\mathrm{C} 3$ » à savoir la plupart des végétaux comestibles d'Europe Occidentale $\left(-33 \% \%_{0}<\delta^{13} \mathrm{C}<-22 \%\right.$ [45]) des végétaux « $\mathrm{C} 4$ » comme, dans cette région, le millet $\left(-16,0 \%{ }_{0}<\delta^{13} \mathrm{C}<-9 \%\right.$ [45]) ;

- les végétaux terrestres $\left(-22,5 \% 0<\delta^{13} \mathrm{C}<-11,9 \%\right.$ [46]) des végétaux marins ($19,6 \%<\delta^{13} \mathrm{C}<-9,6 \%$ [46]) ;

- les végétaux de milieu fermé des végétaux de milieu ouvert [47]. 
$\mathrm{Au}$ niveau du collagène, les compositions isotopiques de l'azote permettent de déterminer la position trophique d'un individu en raison du fractionnement isotopique entre l'alimentation et le consommateur [42]. Ainsi, plus le niveau trophique du consommateur (herbivore < omnivore < carnivore) est élevé et plus ses valeurs de $\delta^{15} \mathrm{~N}$ sont élevées. De même, les chaînes alimentaires plus longues en milieu aquatique expliquent les valeurs plus élevées de $\delta^{15} \mathrm{~N}$ dans les tissus des consommateurs des ressources marines ou d'eau douce [46]. Enfin, les plantes fixatrices d'azote, comme les légumineuses, se distinguent des autres végétaux avec des valeurs comprises entre -2 et $2 \%$ contre entre 0 et $6 \%$ pour les autres végétaux [47].

Majoritairement réalisées sur l'os, les analyses isotopiques permettent de cerner l'alimentation «moyenne » des dix dernières années de la vie des individus adultes en raison du renouvellement perpétuel de la trame osseuse [40]. Parfois, les analyses sont réalisées sur le tissu dentaire. Ce tissu ne subissant pas de renouvellement [48], elles permettent, dans ce cas, de cerner l'alimentation présente au moment de la formation de ces tissus, soit durant l'enfance des individus [38,39]. Ainsi, l'analyse conjointe des signatures isotopiques des tissus dentaires et osseux chez l'adulte, permet de suivre la modification des tendances alimentaires au cours de la vie d'un même un individu.

En conclusion, ces marqueurs permettent d'obtenir une information complète sur l'alimentation des populations passées et sur son évolution au cours de la vie des individus. Dans le cas d'Amiens, et de l'hypothèse d'une origine particulière des individus inhumés, la possibilité de caractériser l'environnement d'origine des ressources alimentaires ainsi que la possibilité de pouvoir comparer les données concernant l'enfance et l'âge adulte est particulièrement appréciable.

\section{$\underline{\text { Extraction du collagène et de la bioapatite et analyses spectrométriques }}$}

Le collagène a été extrait selon le protocole de Longin [49] modifié par Bocherens et al. [50]. Les mesures isotopiques ont été réalisées à l'aide d'un spectromètre de masse isotopique Europa Scientific 20-20 couplé à un analyseur automatisé Europa Scientific. Le standard interne utilisé est du foie de bœuf (IA-R042) dont les teneurs isotopiques sont calibrées à partir des standard de comparaison inter-laboratoire IAEA-CH-6 (sucrose, $\delta^{13} \mathrm{C}=$ $10,43 \%$ ) et IAEA-N-1 (sulfate d'ammonium, $\delta^{15} \mathrm{~N}=0,40 \%$ ). La précision analytique obtenue à partir de l'analyse répétée du standard interne du laboratoire est de $0,05 \%$ pour les valeurs de $\delta^{13} \mathrm{C}$ et de $0,06 \%$ pour celles de $\delta^{15} \mathrm{~N}$. 
La bioapatite est extraite suivant le protocole défini par Balasse et al. [51]. Les mesures isotopiques de la bioapatite ont été réalisées à l'aide d'un spectromètre de masse isotopique Europa Scientific. Le standard interne utilisé au cours de l'analyse est du carbonate de calcium (IA-R022). La précision analytique obtenue à partir de l'analyse répétée du standard interne du laboratoire est de $0,14 \%$ pour les valeurs de $\delta^{13} \mathrm{C}$.

\section{Détermination de l'état de préservation des échantillons}

Les indicateurs considérés pour s'assurer de l'intégrité biochimique du collagène sont : le rendement d'extraction du collagène $\left(\mathrm{Rdt}_{\mathrm{co}},>10 \mathrm{mg} / \mathrm{g}\right.$ [52]), le rapport atomique $\mathrm{du}$ carbone sur l'azote des échantillons $(2,9<\mathrm{C} / \mathrm{N}<3,6$ [53]) et les quantités de carbone $(\% \mathrm{C}$, $>30 \%)$ et d'azote $(\% \mathrm{~N},>10 \%)$ présentes dans chaque échantillon [52,54]. L'indépendance des valeurs de $\delta^{13} \mathrm{C}$ et $\delta^{15} \mathrm{~N}$ avec celles des indicateurs de préservation est également testée statistiquement.

Il n'existe à l'heure actuelle aucun indicateur permettant de s'assurer de manière certaine de l'intégrité biochimique de la bioapatite [55,56]. Dans le cadre de cette étude, il a été choisi de tester statistiquement l'indépendance des résultats de $\delta^{13} \mathrm{C}_{\mathrm{ap}}$ vis-à-vis des éléments de contrôle à notre disposition que sont le rendement d'extraction de l'apatite $\left(\mathrm{Rdt}_{\mathrm{ap}}\right)$, le poids de l'échantillon analysé (Pds), le volume de $\mathrm{CO}_{2}$ libéré $\left(\mathrm{Vol} \mathrm{CO}_{2}\right)$, la quantité de l'équivalent carbonates de calcium contenu dans l'échantillon d'apatite analysé (\% $\left.\mathrm{CO}_{3}\right)$.

\section{$\underline{\text { Modèles interprétatifs }}$}

Afin de préciser les informations relatives aux régimes alimentaires, deux modèles graphiques mis au point à partir de données expérimentales sur des rats, souris et cochons sous régime contrôlé, ont été utilisés [43,44,57,58].

Le premier modèle dit «mono-isotopique» repose sur les valeurs isotopiques du carbone de l'apatite et du collagène $\left(\delta^{13} \mathrm{C}_{\mathrm{ap}}\right.$ et $\left.\delta^{13} \mathrm{C}_{\mathrm{co}}\right)$ [43,44,57]. La projection des valeurs isotopiques des individus relativement à deux droites de régression, correspondant à des régimes alimentaires fondés sur des protéines $\mathrm{C} 3$ ou $\mathrm{C} 4 /$ marines obtenues d'après les données expérimentales, permet de quantifier la part protéinique du régime et d'apprécier la contribution relative de ressources $\mathrm{C} 3$ ou $\mathrm{C} 4$ dans le régime global.

Le second modèle «multi-isotopique » [57] intègre au modèle précédant les valeurs de $\delta^{15} \mathrm{~N}$ afin de discriminer les régimes basés sur les ressources marines de ceux basés sur des 
ressources C4. Les trois variables isotopiques sont « aplaties » en deux fonctions linéaires afin de permettre une représentation graphique simple. Leurs formules sont :

$$
\begin{aligned}
& \mathrm{F} 1=\left(0,322 \cdot \delta^{13} \mathrm{C}_{\mathrm{ap}}\right)+\left(0,727 \cdot \delta^{13} \mathrm{C}_{\mathrm{co}}\right)+\left(0,219 \cdot \delta^{15} \mathrm{~N}\right)+9,354 \\
& \mathrm{~F} 2=\left(-0,393 \cdot \delta^{13} \mathrm{C}_{\mathrm{ap}}\right)+\left(0,133 \cdot \delta^{13} \mathrm{C}_{\mathrm{co}}\right)+\left(0,622 \cdot \delta^{15} \mathrm{~N}\right)-8,703 .
\end{aligned}
$$

La première fonction est plus influencée par les valeurs isotopiques du carbone $\left(\delta^{13} \mathrm{C}_{\mathrm{co}}\right.$ et $\left.\delta^{13} \mathrm{C}_{\mathrm{ap}}\right)$ tandis que la seconde est plus fortement liée à celles de l'azote $\left(\delta^{15} \mathrm{~N}\right)$ [57]. Ce modèle permet d'inférer sur la nature isotopique de l'alimentation protéinique et globale des individus (intégrant en plus la part énergétique de l'alimentation) en fonction de leur position relativement à cinq clusters (définis à partir des valeurs isotopiques de populations préhistoriques d'Amérique du Nord) renvoyant aux régimes suivants :

- régime global $=100 \% \mathrm{C} 3$, régime protéinique $=100 \% \mathrm{C} 3$;

- régime global $=70 \% \mathrm{C} 4$, régime protéinique $>50 \% \mathrm{C} 4$;

- régime global $=50 \% \mathrm{C} 3$, régime protéinique $=$ marin ;

- régime global $=70 \% \mathrm{C} 3$, régime protéinique $\geq 65 \% \mathrm{C} 3$; et (5) régime global $=70 \% \mathrm{C} 4$, régime protéinique $\geq 65 \% \mathrm{C} 3$.

\section{Outils statistiques}

Toutes les analyses statistiques ont été réalisées à l'aide du logiciel R 3.2.2. Le test employé pour la comparaison de deux séries est le test non paramétrique exact de Wilcoxon dans ses deux versions pour variables appariées et variables indépendantes (H0 : les deux séries ont des médianes significativement non différentes, hypothèse acceptée si la valeur de $p$ est supérieure à 0,05). Pour la comparaison d'un nombre de séries supérieures à deux, le test de Kruskal-Wallis dans sa version approximative (Monte Carlo, 10000 réplicats) avec une correction FWER lors des comparaisons deux à deux a été utilisé (H0 : les séries ont des médianes significativement non différentes, hypothèse acceptée si la valeur de $\mathrm{p}$ est supérieure à 0,05 ). L'indépendance entre deux séries a été analysée avec le test de corrélation des rangs de Spearman (H0 : les séries ne sont pas corrélées, hypothèse acceptée si la valeur de $\mathrm{p}$ est supérieure à 0,05 . La corrélation est considérée comme forte si rho >0,6).

\section{RESULTATS}

\section{Etat de préservation des échantillons}


Les échantillons de collagène osseux des humains (Tableau 1) présentent des rendements compris entre 29,1 et 194,4 mg. $\mathrm{g}^{-1}\left(121,2 \pm 51,3 \mathrm{mg} \cdot \mathrm{g}^{-1}, \mathrm{n}=33\right)$, des quantités de carbone comprises entre 31,2 et $44,2 \%(39,5 \pm 2,8 \%, n=33)$, des quantités d'azote comprises entre 11,3 et $16,0 \%(14,4 \pm 1,0 \%, \mathrm{n}=33)$ et des rapports $\mathrm{C} / \mathrm{N}$ entre 3,0 et 3,2 $(3,2 \pm 0,0, \mathrm{n}=33)$. Les échantillons de dentine ont des rendements compris entre 55,0 et $197,6 \mathrm{mg} \cdot \mathrm{g}^{-1}\left(147,4 \pm 32,6 \mathrm{mg} \cdot \mathrm{g}^{-1}, \mathrm{n}=33\right)$, des quantités de carbone et d'azote comprises respectivement entre 33,3 et $41,6 \%(38,5 \pm 2,0 \%, \mathrm{n}=33)$ et entre 12,0 et $15,1 \%$ $(14,0 \pm 0,7 \%, \mathrm{n}=33)$ et enfin des rapports $\mathrm{C} / \mathrm{N}$ comprises entre 3,2 et $3,3(3,2 \pm 0,0, \mathrm{n}=33$; Tableau 1).

Les échantillons de faune (Tableau 3) ont quant à eux des rendements compris entre 12,6 et $177,4 \mathrm{mg} \cdot \mathrm{g}^{-1}\left(90,5 \pm 53,2 \mathrm{mg} \cdot \mathrm{g}^{-1}, \mathrm{n}=28\right)$, des quantités de carbone et d'azote respectivement comprises entre 28,9 et $45,1 \%(40,6 \pm 3,2 \%, \mathrm{n}=28)$ et entre 10,9 et $16,6 \%$ $(14,9 \pm 1,1 \%, \mathrm{n}=28)$ et des rapports $\mathrm{C} / \mathrm{N}$ compris entre 3,1 et $3,3(3,2 \pm 0,0, \mathrm{n}=28)$.

D'après les critères définis ci-dessus, l'intégralité des échantillons de collagène peut être considérée comme bien conservé et non contaminé ce que confirment les tests de corrélations de rangs de Spearman ne montrant aucune corrélation forte significative (à savoir valeur de $\mathrm{p}<0,05$ et rho $>0,6$ Tableau 4) entre les quatre paramètres retenus $\left(\mathrm{Rdt}_{\mathrm{co}}, \mathrm{C} / \mathrm{N}, \% \mathrm{C}\right.$ et $\% \mathrm{~N}$ ) et les valeurs isotopiques.

Les rendements de purification de la bioapatite des échantillons d'émail sont compris entre 42,3 et $69,8 \%(58,2 \pm 7,5 \%, \mathrm{n}=33)$ ce qui correspond à des échantillons d'apatite pesant entre 2,7 et $6,1 \mathrm{mg}(4,8 \pm 0,9 \mathrm{mg}, \mathrm{n}=33)$. Le volume de $\mathrm{CO}_{2}$ dégagé lors de leur analyse est compris entre 0,024 et $0,061 \mathrm{ml}$ et la quantité de carbonates $\left(\mathrm{CO}_{3}\right)$ est comprise entre 3,6 et $6,1 \%(4,5 \pm 0,6 \%, \mathrm{n}=33$; TABLEAU 2). Pour les échantillons d'os, les rendements sont compris entre 22,2 et 53,4\% $(36,9 \pm 9,0 \%, \mathrm{n}=33)$ pour des échantillons analysés pesant entre 2,7 et $8,9 \mathrm{mg}(6,5 \pm 1,5 \mathrm{mg}, \mathrm{n}=33)$. Le volume de $\mathrm{CO}_{2}$ dégagé varie entre 0,037 et $0,154 \mathrm{ml}(0,072 \pm 0,0252 \mathrm{ml}, \mathrm{n}=33)$ et la quantité de l'équivalent carbonates de calcium $\left(\mathrm{CO}_{3}\right)$ est comprise entre 2,6 et $10,4 \%(5,1 \pm 1,8 \%, \mathrm{n}=33$;Tableau 2).

Les résultats des tests de corrélations entre les paramètres disponibles et les valeurs isotopiques des échantillons d'apatite ne montrent aucune corrélation forte significative (pour rappel, valeur de $\mathrm{p}<0,05$ et rho $>0,6$, Tableau 4). Les échantillons (os comme émail) peuvent donc tous être considérés comme bien conservés.

\section{$\underline{\text { Valeurs isotopiques }}$}


Les valeurs de $\delta^{13} \mathrm{C}$ de l'ensemble de la faune varient entre $-22,7$ et $-20,0 \%$ $(-21,7 \pm 0,7 \%, \mathrm{n}=28)$ tandis que celles de $\delta^{15} \mathrm{~N}$ varient entre 2,8 et $10,0 \%$ o $(6,4 \pm 1,6 \%$, $\mathrm{n}=28$ ) (Tableau 3,Erreur ! Source du renvoi introuvable.). Les valeurs de $\delta^{13} \mathrm{C}$ des trois groupes d'herbivores, (B. taurus, E. caballus, O. aries) sont statistiquement comparables (Tableau 5) et sont comprises entre $-22,7$ et $-21,3 \%$ o $(-22,1 \pm 0,5 \%, \mathrm{n}=16)$. Celles de $\delta^{15} \mathrm{~N}$ sont également statistiquement comparables (Tableau ) et sont comprises entre 2,8 et 8,9\%o $(6,5 \pm 1,6 \%, \mathrm{n}=15)$. Les Sus scrofa ont des valeurs de $\delta^{13} \mathrm{C}$ comprises entre $-21,5$ et $-20,6 \%$ o $(-21,3 \pm 0,3 \%, \mathrm{n}=10)$ et celles de $\delta^{15} \mathrm{~N}$ entre 4,7 et $6,0 \%$ o $(5,6 \pm 0,4 \%, \mathrm{n}=10)$. Les valeurs de $\delta^{13} \mathrm{C}$ du Canis familiaris et du Gallus sp. sont respectivement de -20,0 \% et $-20,7 \%$ et celles de $\delta^{15} \mathrm{~N}$ de $9,4 \%$ et $10,0 \%$. Les Sus scrofa $(\mathrm{n}=10)$ ont des valeurs de $\delta^{15} \mathrm{~N}$ statistiquement inférieures à celles des herbivores $(\mathrm{n}=16)$ mais des valeurs de $\delta^{13} \mathrm{C}$ statistiquement plus élevées (Tableau 6).

Pour les sujets humains, au niveau de l'os, les valeurs de $\delta^{13} \mathrm{C}_{\mathrm{co}}$ sont comprises entre $-20,4$ et $-15,3 \%$ o $(-19,4 \pm 0,8 \% ; n=33)$, celles de $\delta^{15} \mathrm{~N}$ entre 7,4 et $12,3 \%$ o $(9,4 \pm 1,0 \%$, $\mathrm{n}=33)$ et celles de $\delta^{13} \mathrm{C}_{\mathrm{ap}}$ entre $-14,9$ et $-11,3 \%$ o $(-13,6 \pm 0,8 \%$, $\mathrm{n}=33$; Tableau 1,TABLEAU 2,Erreur ! Source du renvoi introuvable.,FIG.Erreur ! Source du renvoi introuvable.,FIG.Erreur ! Source du renvoi introuvable.). Au niveau des racines dentaires, les valeurs de $\delta^{13} \mathrm{C}_{\mathrm{co}}$ sont comprises entre $-20,2$ et $-15,1 \%$ o $(-19,5 \pm 0,8 \%$, $\mathrm{n}=33)$, celles de $\delta^{15} \mathrm{~N}$ entre 7,4 et $12,3 \%(9,4 \pm 1,0 \%, \mathrm{n}=33$ TABLEAU 1, ,Erreur ! Source du renvoi introuvable.). Au niveau de la couronne les valeurs de $\delta^{13} \mathrm{C}_{\mathrm{ap}}$ sont comprises entre $-15,8$ et $-10,9 \%$ \% $(-13,9 \pm 0,8 \%, n=33$; TABLEAU 1,TABLEAU 2,Erreur ! Source du renvoi introuvable.). Aucune différence n'est statistiquement décelable entre les valeurs de $\delta^{13} \mathrm{C}_{\mathrm{co}}$ et $\delta^{13} \mathrm{C}_{\mathrm{ap}}$ des os et des dents (

Tableau ). Les valeurs de $\delta^{15} \mathrm{~N}$ des dents sont significativement supérieures à celles des os (

Tableau ).

Les valeurs de $\delta^{13} \mathrm{C}_{\mathrm{co}}$, de $\delta^{15} \mathrm{~N}$ et $\delta^{13} \mathrm{C}_{\mathrm{ap}}$ des sujets masculins et des sujets féminins (Tableau 4-Résultats des tests de corrélation de rang de Spearman - Spearman correlation tests results

\begin{tabular}{lcccc}
\hline & Facteur considéré & $\mathbf{S}$ & rho & p-value \\
\hline $\boldsymbol{\delta}^{\mathbf{1 3}} \mathbf{C}_{\text {faune }}$ & $\mathrm{Rdt}_{\mathrm{co}}$ & 1944,5 & 0,47 & $\mathbf{0 , 0 1}$ \\
& $\% \mathrm{C}$ & 3902,6 & $-0,07$ & 0,73 \\
& $\% \mathrm{~N}$ & 3974,1 & $-0,09$ & 0,66 \\
$\boldsymbol{\delta}^{\mathbf{1 5}} \mathbf{N}_{\text {faune }}$ & $\mathrm{C} / \mathrm{N}$ & 2196,2 & 0,40 & $\mathbf{0 , 0 4}$ \\
& $\mathrm{Rdt}_{\mathrm{co}}$ & 3561,0 & 0,03 & 0,90
\end{tabular}




\begin{tabular}{|c|c|c|c|c|}
\hline & $\% \mathrm{C}$ & 2349,5 & 0,36 & 0,06 \\
\hline & $\% \mathrm{~N}$ & 2490,8 & 0,32 & 0,10 \\
\hline \multirow{6}{*}{$\delta^{13} \mathbf{C}_{\mathrm{co} / \mathrm{ss}}$} & $\mathrm{C} / \mathrm{N}$ & 4125,6 & $-0,13$ & 0,51 \\
\hline & $\mathrm{Rdt}_{\mathrm{co}}$ & 5592,8 & 0,07 & 0,72 \\
\hline & $\% \mathrm{C}$ & 5911,4 & 0,01 & 0,95 \\
\hline & $\% \mathrm{~N}$ & 5829,2 & 0,03 & 0,89 \\
\hline & $\mathrm{C} / \mathrm{N}$ & 6160,6 & $-0,03$ & 0,87 \\
\hline & Age & 4261,4 & 0,29 & 0,10 \\
\hline \multirow[t]{5}{*}{$\delta^{15} \mathbf{N}_{\mathrm{os}}$} & $\mathrm{Rdt}_{\mathrm{co}}$ & 4725,9 & 0,21 & 0,24 \\
\hline & $\% \mathrm{C}$ & 4594,1 & 0,23 & 0,19 \\
\hline & $\% \mathrm{~N}$ & 4952,6 & 0,17 & 0,34 \\
\hline & $\mathrm{C} / \mathrm{N}$ & 3505,2 & 0,41 & 0,02 \\
\hline & Age & 7035,1 & $-0,18$ & 0,33 \\
\hline \multirow[t]{5}{*}{$\delta^{13} \mathbf{C}_{\mathrm{ap} / \mathrm{os}}$} & $\mathrm{Rdt}_{\mathrm{ap}}$ & 7413,2 & $-0,24$ & 0,18 \\
\hline & Pds & 6155,0 & $-0,03$ & 0,87 \\
\hline & $\mathrm{Vol} \mathrm{CO}$ & 3363,5 & 0,44 & 0,01 \\
\hline & $\% \mathrm{CO}_{3}$ & 3539,2 & 0,41 & 0,02 \\
\hline & Age & 6798,5 & $-0,14$ & 0,45 \\
\hline \multirow[t]{5}{*}{$\delta^{13} \mathrm{C}_{\mathrm{co} / \mathrm{dt}}$} & $\mathrm{Rdt}_{\mathrm{co}}$ & 3703,5 & 0,38 & $\mathbf{0 , 0 3}$ \\
\hline & $\% \mathrm{C}$ & 4266,6 & 0,29 & 0,11 \\
\hline & $\% \mathrm{~N}$ & 4104,8 & 0,31 & 0,08 \\
\hline & $\mathrm{C} / \mathrm{N}$ & 7063,8 & $-0,18$ & 0,31 \\
\hline & Age & 4393,2 & 0,27 & 0,13 \\
\hline \multirow[t]{5}{*}{$\delta^{15} \mathbf{N}_{\mathrm{dt}}$} & $\mathrm{Rdt}_{\mathrm{co}}$ & 5457,9 & 0,09 & 0,63 \\
\hline & $\% \mathrm{C}$ & 7212,7 & $-0,21$ & 0,25 \\
\hline & $\% \mathrm{~N}$ & 7041,4 & $-0,18$ & 0,33 \\
\hline & $\mathrm{C} / \mathrm{N}$ & 4962,8 & 0,17 & 0,34 \\
\hline & Age & 7481,9 & $-0,25$ & 0,16 \\
\hline \multirow{5}{*}{$\delta^{13} \mathbf{C}_{\mathrm{ap} / \mathrm{dt}}$} & $\mathrm{Rdt}_{\mathrm{ap}}$ & 4726,8 & 0,21 & 0,24 \\
\hline & $\mathrm{Pds}$ & 5424,0 & 0,09 & 0,60 \\
\hline & $\mathrm{Vol} \mathrm{CO} 2$ & 5222,7 & 0,13 & 0,48 \\
\hline & $\% \mathrm{CO}_{3}$ & 5777,9 & 0,03 & 0,85 \\
\hline & & 5728,9 & 0,04 & 0,81 \\
\hline
\end{tabular}

Résultats significatifs en gras, corrélation par convention comme forte seulement si rho $>0,6$ 
Tableau 5-Résultats des tests approximatifs (Monte Carlo, 10000 réplicats) de Kruskal Wallis - Approximative Kruskal Wallis test results (Monte Carlo, 10000 replicats)

\begin{tabular}{lcc}
\hline & Chi $^{2}$ & p-value \\
\hline $\begin{array}{l}\text { Bos taurus }(\mathbf{n = 1 1}), \text { Equus caballus }(\mathbf{n}=\mathbf{2}), \\
\text { Ovis aries }(\mathbf{n = 3})\end{array}$ & \\
$\delta^{13} \mathrm{C}$ & 4,412 & 0,12 \\
$\delta^{15} \mathrm{~N}$ & 3,277 & 0,21 \\
Secteurs $\mathbf{1}(\mathbf{n = 1}), \mathbf{2}(\mathbf{n = 2}), \mathbf{3}(\mathbf{n = 3}), \mathbf{4}(\mathbf{n}=\mathbf{9})$, & & \\
$\mathbf{5}(\mathbf{n = 1 5}), \mathbf{6}(\mathbf{n = 2})$ et hors secteur $(\mathbf{n = 1})$ & & \\
$\delta^{13} \mathrm{C}_{\mathrm{co} / \mathrm{os}}$ & 11,528 & 0,19 \\
$\delta^{15} \mathrm{~N}_{\mathrm{os}}$ & 7,858 & 0,26 \\
$\delta^{13} \mathrm{C}_{\mathrm{ap} / \mathrm{os}}$ & 10,615 & 0,39 \\
$\delta^{13} \mathrm{C}_{\mathrm{co} / \mathrm{dt}}$ & 6,632 & 0,35 \\
$\delta^{15} \mathrm{~N}_{\mathrm{dt}}$ & 7,383 & 0,61 \\
$\delta^{13} \mathrm{C}_{\mathrm{ap} / \mathrm{dt}}$ & 2,892 & 0,88 \\
\hline
\end{tabular}

Tableau 6-Résultats des tests exacts de Wilcoxon bivariés - Wilcoxon exact unpaired tests results

\begin{tabular}{lrr}
\hline & \multicolumn{1}{c}{$\mathbf{Z}$} & p-value \\
\hline Herbivores $(\mathbf{n}=\mathbf{1 6})$, Sus scrofa $(\mathbf{n = 1 0})$ & & \\
$\delta^{13} \mathrm{C}$ (différence $\left.>0\right)$ & 3,8224 & $\mathbf{9 . 4 e - 0 6}$ \\
$\delta^{15} \mathrm{~N}$ (différence <0) & $-1,9768$ & $\mathbf{0 . 0 2}$ \\
Hommes $(\mathbf{n = 1 5})$, femmes $(\mathbf{n = 1 7 )}$ & & \\
$\delta^{13} \mathrm{C}_{\mathrm{co} / \mathrm{os}}$ & 0,5289 & 0,61 \\
$\delta^{15} \mathrm{~N}_{\mathrm{os}}$ & $-0,3777$ & 0,72 \\
$\delta^{13} \mathrm{C}_{\mathrm{ap} / \mathrm{os}}$ & 1,6427 & 0,11 \\
$\delta^{13} \mathrm{C}_{\mathrm{co} / \mathrm{dt}}$ & 0,8312 & 0,42 \\
$\delta^{15} \mathrm{~N}_{\mathrm{dt}}$ & $-0,2455$ & 0,82 \\
$\delta^{13} \mathrm{C}_{\mathrm{ap} / \mathrm{dt}}$ & 0,1511 & 0,89 \\
\hline
\end{tabular}

Tableau 7-Résultats des tests exacts de Wilcoxon appariés- Wilcoxon exact paired tests results

\begin{tabular}{ccc}
\hline & $\mathbf{Z}$ & p value \\
\hline Dents $(\mathbf{n = 3 3})$, os $(\mathbf{n = 3 3})$ & & \\
$\delta^{13} \mathrm{C}_{\mathrm{co}}$ & $-1,644$ & 0,10 \\
$\delta^{15} \mathrm{~N}$ & 3,5827 & $\mathbf{0 , 0 0}$ \\
$\delta^{13} \mathrm{C}_{\mathrm{ap}}$ & 0,83095 & 0,41 \\
\hline
\end{tabular}


Tableau ) ne sont pas statistiquement différentes quel que soit le tissu analysé (Tableau ). De la même manière, les valeurs de $\delta^{13} \mathrm{C}_{\mathrm{co}}$, de $\delta^{15} \mathrm{~N}$ et $\delta^{13} \mathrm{C}_{\mathrm{ap}}$ de l'os comme de l'émail des individus échantillonnés ne présentent pas de différences statistiques lorsque c'est le secteur d'inhumation qui est pris en considération (Tableau 4-Résultats des tests de corrélation de rang de Spearman - Spearman correlation tests results

\begin{tabular}{|c|c|c|c|c|}
\hline & Facteur considéré & $\mathbf{S}$ & rho & p-value \\
\hline \multirow[t]{4}{*}{$\delta^{13} C_{\text {faune }}$} & $\mathrm{Rdt}_{\mathrm{co}}$ & 1944,5 & 0,47 & $\mathbf{0 , 0 1}$ \\
\hline & $\% \mathrm{C}$ & 3902,6 & $-0,07$ & 0,73 \\
\hline & $\% \mathrm{~N}$ & 3974,1 & $-0,09$ & 0,66 \\
\hline & $\mathrm{C} / \mathrm{N}$ & 2196,2 & 0,40 & 0,04 \\
\hline \multirow[t]{4}{*}{$\delta^{15} \mathbf{N}_{\text {faune }}$} & $\mathrm{Rdt}_{\mathrm{co}}$ & 3561,0 & 0,03 & 0,90 \\
\hline & $\% \mathrm{C}$ & 2349,5 & 0,36 & 0,06 \\
\hline & $\% \mathrm{~N}$ & 2490,8 & 0,32 & 0,10 \\
\hline & $\mathrm{C} / \mathrm{N}$ & 4125,6 & $-0,13$ & 0,51 \\
\hline \multirow{5}{*}{$\delta^{13} \mathrm{C}_{\mathrm{co} / \mathrm{os}}$} & $\mathrm{Rdt}_{\mathrm{co}}$ & 5592,8 & 0,07 & 0,72 \\
\hline & $\% \mathrm{C}$ & 5911,4 & 0,01 & 0,95 \\
\hline & $\% \mathrm{~N}$ & 5829,2 & 0,03 & 0,89 \\
\hline & $\mathrm{C} / \mathrm{N}$ & 6160,6 & $-0,03$ & 0,87 \\
\hline & Age & 4261,4 & 0,29 & 0,10 \\
\hline \multirow[t]{5}{*}{$\delta^{15} \mathbf{N}_{\mathrm{os}}$} & $\mathrm{Rdt}_{\mathrm{co}}$ & 4725,9 & 0,21 & 0,24 \\
\hline & $\% \mathrm{C}$ & 4594,1 & 0,23 & 0,19 \\
\hline & $\% \mathrm{~N}$ & 4952,6 & 0,17 & 0,34 \\
\hline & $\mathrm{C} / \mathrm{N}$ & 3505,2 & 0,41 & $\mathbf{0 , 0 2}$ \\
\hline & Age & 7035,1 & $-0,18$ & 0,33 \\
\hline \multirow[t]{5}{*}{$\delta^{13} C_{a p / o s}$} & $\mathrm{Rdt}_{\mathrm{ap}}$ & 7413,2 & $-0,24$ & 0,18 \\
\hline & Pds & 6155,0 & $-0,03$ & 0,87 \\
\hline & Vol CO 2 & 3363,5 & 0,44 & $\mathbf{0 , 0 1}$ \\
\hline & $\% \mathrm{CO}_{3}$ & 3539,2 & 0,41 & $\mathbf{0 , 0 2}$ \\
\hline & Age & 6798,5 & $-0,14$ & 0,45 \\
\hline \multirow[t]{5}{*}{$\delta^{13} \mathrm{C}_{\mathrm{co} / \mathrm{dt}}$} & $\mathrm{Rdt}_{\mathrm{co}}$ & 3703,5 & 0,38 & $\mathbf{0 , 0 3}$ \\
\hline & $\% \mathrm{C}$ & 4266,6 & 0,29 & 0,11 \\
\hline & $\% \mathrm{~N}$ & 4104,8 & 0,31 & 0,08 \\
\hline & $\mathrm{C} / \mathrm{N}$ & 7063,8 & $-0,18$ & 0,31 \\
\hline & Age & 4393,2 & 0,27 & 0,13 \\
\hline \multirow[t]{5}{*}{$\delta^{15} \mathbf{N}_{\mathrm{dt}}$} & $\mathrm{Rdt}_{\mathrm{co}}$ & 5457,9 & 0,09 & 0,63 \\
\hline & $\% \mathrm{C}$ & 7212,7 & $-0,21$ & 0,25 \\
\hline & $\% \mathrm{~N}$ & 7041,4 & $-0,18$ & 0,33 \\
\hline & $\mathrm{C} / \mathrm{N}$ & 4962,8 & 0,17 & 0,34 \\
\hline & Age & 7481,9 & $-0,25$ & 0,16 \\
\hline \multirow[t]{5}{*}{$\delta^{13} \mathrm{C}_{\mathrm{ap} / \mathrm{dt}}$} & $\mathrm{Rdt}_{\mathrm{ap}}$ & 4726,8 & 0,21 & 0,24 \\
\hline & $\mathrm{Pds}$ & 5424,0 & 0,09 & 0,60 \\
\hline & $\mathrm{Vol} \mathrm{CO}_{2}$ & 5222,7 & 0,13 & 0,48 \\
\hline & $\% \mathrm{CO}_{3}$ & 5777,9 & 0,03 & 0,85 \\
\hline & Age & 5728,9 & 0,04 & 0,81 \\
\hline
\end{tabular}

Résultats significatifs en gras, corrélation par convention comme forte seulement si rho $>0,6$ 
Tableau 5-Résultats des tests approximatifs (Monte Carlo, 10000 réplicats) de Kruskal Wallis - Approximative Kruskal Wallis test results (Monte Carlo, 10000 replicats)

\begin{tabular}{lcc}
\hline \multicolumn{2}{l}{ Chi $^{\mathbf{2}}$} & p-value \\
\hline $\begin{array}{l}\text { Bos taurus }(\mathbf{n = 1 1}), \text { Equus caballus }(\mathbf{n}=\mathbf{2}), \\
\text { Ovis aries }(\mathbf{n = 3})\end{array}$ & \\
$\delta^{13} \mathrm{C}$ & 4,412 & 0,12 \\
$\delta^{15} \mathrm{~N}$ & 3,277 & 0,21 \\
Secteurs $\mathbf{1}(\mathbf{n = 1}), \mathbf{2}(\mathbf{n = 2}), \mathbf{3}(\mathbf{n = 3}), \mathbf{4}(\mathbf{n}=\mathbf{9})$, & & \\
$\mathbf{5}(\mathbf{n}=\mathbf{1 5}), \mathbf{6}(\mathbf{n = 2})$ et hors secteur $(\mathbf{n = 1})$ & & \\
$\delta^{13} \mathrm{C}_{\mathrm{co} / \mathrm{os}}$ & 11,528 & 0,19 \\
$\delta^{15} \mathrm{~N}_{\mathrm{os}}$ & 7,858 & 0,26 \\
$\delta^{13} \mathrm{C}_{\mathrm{ap} / \mathrm{os}}$ & 10,615 & 0,39 \\
$\delta^{13} \mathrm{C}_{\mathrm{co} / \mathrm{dt}}$ & 6,632 & 0,35 \\
$\delta^{15} \mathrm{~N}_{\mathrm{dt}}$ & 7,383 & 0,61 \\
$\delta^{13} \mathrm{C}_{\mathrm{ap} / \mathrm{dt}}$ & 2,892 & 0,88 \\
\hline
\end{tabular}

Tableau 6-Résultats des tests exacts de Wilcoxon bivariés - Wilcoxon exact unpaired tests results

\begin{tabular}{lrr}
\hline & \multicolumn{1}{c}{$\mathbf{Z}$} & p-value \\
\hline Herbivores $(\mathbf{n}=\mathbf{1 6})$, Sus scrofa $(\mathbf{n = 1 0})$ & & \\
$\delta^{13} \mathrm{C}$ (différence $\left.>0\right)$ & 3,8224 & $\mathbf{9 . 4 e - 0 6}$ \\
$\delta^{15} \mathrm{~N}$ (différence <0) & $-1,9768$ & $\mathbf{0 . 0 2}$ \\
Hommes $(\mathbf{n = 1 5})$, femmes $(\mathbf{n = 1 7 )}$ & & \\
$\delta^{13} \mathrm{C}_{\mathrm{co} / \mathrm{os}}$ & 0,5289 & 0,61 \\
$\delta^{15} \mathrm{~N}_{\mathrm{os}}$ & $-0,3777$ & 0,72 \\
$\delta^{13} \mathrm{C}_{\mathrm{ap} / \mathrm{os}}$ & 1,6427 & 0,11 \\
$\delta^{13} \mathrm{C}_{\mathrm{co} / \mathrm{dt}}$ & 0,8312 & 0,42 \\
$\delta^{15} \mathrm{~N}_{\mathrm{dt}}$ & $-0,2455$ & 0,82 \\
$\delta^{13} \mathrm{C}_{\mathrm{ap} / \mathrm{dt}}$ & 0,1511 & 0,89 \\
\hline
\end{tabular}

Tableau 7-Résultats des tests exacts de Wilcoxon appariés- Wilcoxon exact paired tests results

\begin{tabular}{ccc}
\hline & $\mathbf{Z}$ & $\mathbf{p}$ value \\
\hline Dents $(\mathbf{n}=\mathbf{3 3})$, os $(\mathbf{n = 3 3})$ & & \\
$\delta^{13} \mathrm{C}_{\mathrm{co}}$ & $-1,644$ & 0,10 \\
$\delta^{15} \mathrm{~N}$ & 3,5827 & $\mathbf{0 , 0 0}$ \\
$\delta^{13} \mathrm{C}_{\mathrm{ap}}$ & 0,83095 & 0,41 \\
\hline
\end{tabular}


Tableau ,Tableau ).

En ce qui concerne l'âge au décès, les résultats des tests de corrélation de Spearman ne sont pas significatifs (Tableau ).

\section{DISCUSSION}

\section{$\underline{\text { L'alimentation des habitants d'Amiens }}$}

Les valeurs de $\delta^{13} \mathrm{C}_{\mathrm{co}}$ des animaux sont cohérentes avec un environnement tempéré ouvert dominé par les végétaux à photosynthèse de type C3 [46] (FIG.2). La variabilité des valeurs isotopiques de $\delta^{15} \mathrm{~N}$ au sein de l'échantillon d'herbivores est conforme à celle observée chez les herbivores terrestres dans un tel environnement [59]. Le Canis familiaris comme le Gallus sp. présentent des valeurs de $\delta^{13} \mathrm{C}$ et de $\delta^{15} \mathrm{~N}$ équivalentes à celles des individus humains et semblent donc se nourrir des restes alimentaires de ceux-ci.

Comparativement aux signatures isotopiques des herbivores, les porcs présentent des valeurs de $\delta^{15} \mathrm{~N}$ plus faibles et des valeurs de $\delta^{13} \mathrm{C}$ plus élevées. Ils semblent donc avoir un régime alimentaire certes herbivore mais centré sur d'autres ressources végétales enrichies en ${ }^{13} \mathrm{C}$, comme par exemple les glands ou les champignons [60] et appauvries en ${ }^{15} \mathrm{~N}$, comme le sont les légumineuses. Ceci est cohérent avec la documentation historique de pratiques d'élevage spécifiques des porcs [61].

Les hommes et les femmes d'Amiens possèdent des régimes alimentaires équivalents (TABLEAU 6,TABLEAU 8), comme c'est le cas dans d'autres sites, datés de la même période, en Angleterre [12,20] ou en Tunisie [4]. Les résultats des tests de corrélation entre les valeurs isotopiques et l'âge au décès ne montrent aucune tendance alimentaire liée à l'âge au décès chez les adultes (TABLEAU 4). Ce résultat est contraire à ce qui a été mis en évidence dans la nécropole d'Isola Sacra [5]. Enfin, aucune différence significative n'existe entre les valeurs des sujets des différents secteurs d'inhumation (TABLEAU 5). La localisation des tombes au sein de l'aire funéraire ne semble donc pas corrélée à des habitudes culturelles particulières en lien avec l'alimentation.

En conclusion, il semblerait qu'en ce qui concerne l'alimentation, les habitants d'Amiens forment un ensemble homogène sur lequel les facteurs archéologiques et biologiques ne semblent pas avoir d'influence.

En ce qui concerne la définition du régime alimentaire des humains, le modèle monoisotopique (FIG.3) combinant, pour rappel, les valeurs de $\delta^{13} \mathrm{C}_{\mathrm{ap}}$ et $\delta^{13} \mathrm{C}_{\mathrm{co}}$, semble indiquer que les sujets analysés ont un régime protéinique issu d'un environnement $\mathrm{C} 3$. Le régime 
lipido-glucidique semble lui aussi majoritairement constitué de ressources C3 même si la dispersion des valeurs le long de la « ligne des protéines » permet de supposer la contribution, dans une faible proportion, d'une ressource enrichie en ${ }^{13} \mathrm{C}$ comme le millet ou les ressources marines. Cette consommation, si elle est avérée, serait moins importante que celles constatées à Rome [29], Isola Sacra [25], Leptiminus [4] et Rogowo [31] (FIG.3).

Selon le modèle multi-isotopique (FIG.4), les sujets d'Amiens se trouve à la limite des clusters 1 (régime glucides/lipides : $100 \% \mathrm{C} 3$ et régime protéines : $100 \% \mathrm{C} 3$ ) et 4 (régime glucides/lipides : $30 \% \mathrm{C} 4,70 \% \mathrm{C} 3$ et régime protéines : < 35\% C4).

Les populations ayant permis de générer les clusters ont une alimentation très riche en protéines animales notamment du fait de l'exploitation des ressources dulcicoles aux valeurs de $\delta^{15} \mathrm{~N}$ très élevées [62]. Les valeurs de la fonction 2 (reflétant principalement les valeurs de $\left.\delta^{15} \mathrm{~N}\right)$ plus basses des sujets d'Amiens seraient donc le signe d'une alimentation comparativement plus pauvre en protéines animales et ne comprenant pas de ressources d'eau douce. La consommation de ressources $\mathrm{C} 4$ ou de ressources marines dans une faible proportion $(<30 \%)$ expliquerait la proximité des individus avec le cluster 4 dans lequel se retrouvent les sujets de Rogowo [31] et de Rome [29] mais aussi les individus d'Isola Sacra [25] (FIG.4). Dans le cas de cette dernière population, c'est la consommation de ressources marines qui avait été proposée par les auteurs [25]. Le modèle multi-isotopique ne se révèle donc pas performant pour trancher, dans le cas d'une alimentation globalement $\mathrm{C} 3$, entre un apport en ressources marines et la consommation de ressources $\mathrm{C} 4$.

Les conclusions issues de l'étude des modèles interprétatifs (alimentation majoritairement $\mathrm{C} 3$ avec consommation très faible de ressources enrichies en ${ }^{13} \mathrm{C}$ ) sont confirmées par la comparaison entre les valeurs isotopiques de la faune et celles des humains (FIG.2). En effet, l'enrichissement moyen en carbone entre les valeurs de la faune et celles des humains $\left(\Delta^{13} \mathrm{C}_{\text {hum-faune }}=2,2 \%\right.$ ) est supérieur à celui attendu (entre 0 et $2 \%$ [42]) pour la relation trophique simple que laisse supposer l'enrichissement moyen en azote $\left(\Delta^{15} \mathrm{~N}_{\text {hum- }}\right.$ faune $=3,0 \%$, normalement compris entre $3 \%$ et $5 \%$ [41]). Une ressource enrichie en ${ }^{13} \mathrm{C}$ semble donc intervenir dans l'alimentation protéinique. La consommation préférentielle de porc $\left(\Delta^{13} \mathrm{C}_{\text {hum-porc }}=1,8 \%\right.$ et $\Delta \Delta^{15} \mathrm{~N}_{\text {hum-porc }}=3,8 \%$ ) peut être supposée pour une partie de la population mais l'enrichissement en carbone reste quand même relativement élevé pour s'expliquer par une simple alimentation mono-isotopique (entre 0 et $1 \%$ [42]). Il est cependant impossible de trancher entre la consommation de ressources marines ou celle du 
millet, l'exploitation de ces deux ressources étant documentée archéologiquement en Gaule du Nord et à Amiens [6,7,9].

En conclusion, les sujets étudiés possèdent des valeurs isotopiques qui, lorsqu'elles sont mises en rapport avec la faune locale échantillonnée, ne sont pas cohérentes avec une relation simple alimentation-consommateur dans un milieu terrestre de type $\mathrm{C} 3$. Les valeurs isotopiques, qu'elles soient dosées sur le collagène ou l'apatite, permettent de proposer un régime alimentaire global reposant sur (1) une grande majorité de ressources C3 incluant de la viande (herbivores, cochons, volaille), des produits laitiers, des œufs, et des céréales comme le blé, l'orge et l'avoine, et (2) une ressource enrichie en ${ }^{13} \mathrm{C}$, qu'il s'agisse du millet et/ou des ressources marines.

\section{$\underline{\text { Sujets particuliers }}$}

Cinq sujets se distinguent de l'ensemble du groupe par des valeurs isotopiques les plaçant en marge de la variabilité décrite pour ces 33 individus.

Le sujet 2104 (sexe masculin, 46 ans) présente une valeur de $\delta^{13} \mathrm{C}_{\mathrm{co}}(-20,4 \%$ o) relativement basse associée à une valeur de $\delta^{15} \mathrm{~N}(10,0 \%)$ parmi les plus hautes. La valeur de $\delta^{13} \mathrm{C}_{\mathrm{ap}}(-14,9 \%)$ est tout à fait dans la variabilité des autres sujets. La combinaison de ces trois valeurs indiquerait une consommation de ressources dulcicoles (riches en ${ }^{15} \mathrm{~N}$ mais appauvries en ${ }^{13} \mathrm{C}$ [62]). Une telle différence de pratique alimentaire pourrait indiquer soit des préférences alimentaires particulières individuelles, soit une mobilité de cet individu peu avant sa mort.

Le sujet 2113 (sexe féminin, 24 ans) se distingue par des valeurs hautes de $\delta^{13} \mathrm{C}_{\mathrm{co}}$ $\left(-18,7 \%\right.$ ) et de $\delta^{13} \mathrm{C}_{\text {ap }}(-12,7 \%$ ). Ces valeurs renvoient à une consommation de ressources enrichies en ${ }^{13} \mathrm{C}$ (millet ?), à nouveau une préférence alimentaire tout comme une mobilité peu avant la mort peuvent être envisagées.

Le sujet 2023 (sexe masculin, 20 ans) possède une valeur de $\delta^{15} \mathrm{~N}$ extrême $(12,3 \%$ ) qui témoignerait d'une consommation de ressources enrichies en ${ }^{15} \mathrm{~N}$, il pourrait s'agir de ressources marines ou dulcicoles, tout comme de la viande de volailles et de porcs ou bien d'une absence d'aliments appauvris en ${ }^{15} \mathrm{~N}$ (légumineuses) contrairement aux autres individus, ou encore d'un stress physiologique (certains stress ayant tendance à augmenter les valeurs de $\delta^{15} \mathrm{~N}$ [63]). Les valeurs de $\delta^{13} \mathrm{C}$ (co : -19,7\%o; ap : -12,3\%o) s'inscrivant dans la variabilité des autres sujets plaiderait en faveur de l'hypothèse d'un stress ante mortem. 
Le sujet 1033 (sexe féminin, 32 ans) possède une valeur de $\delta^{15} \mathrm{~N}(7,4 \%$ ) très basse qui témoignerait d'une alimentation comprenant peu de protéines animales ou plus de légumineuses en comparaison du reste de la population. Les valeurs de $\delta^{13} \mathrm{C}$ (co : $-19,6 \%$; ap : -14,6\%) s'inscrive dans la variabilité des autres sujets. Ce sujet se distingue également par la localisation de son inhumation, à l'écart du reste de la population. Un régime alimentaire essentiellement herbivore étant généralement associé à un statut social défavorisé [12], la position à l'écart de la sépulture pourrait alors être le reflet d'une situation sociale marginale.

Le sujet 2172 (sexe féminin, 21 ans) est remarquable quelles que soient les valeurs isotopiques considérées $\left(\delta^{13} \mathrm{C}_{\mathrm{co}}-15,3 \% ; \delta^{15} \mathrm{~N} 12,3 \%\right.$; $\left.\delta^{13} \mathrm{C}_{\mathrm{ap}}-11,3 \% \mathrm{\%}\right)$. Elles renvoient à un régime alimentaire fondamentalement différent de celui du reste de la population échantillonnée. Quel que soit le modèle utilisé, ce régime comporte une part non négligeable de protéines marines ou issues d'un réseau trophique de type C4 (FIG 3 et 4). Si une telle particularité paraît plus en faveur d'une origine géographique différente du défunt, qui aurait juste pris dernière demeure à Amiens, rien ne peut exclure une préférence alimentaire individuelle qu'elle relève d'un tabou ou du suivi de certaines règles communautaires, ou encore d'une indisposition physiologique à certains types d'aliments.

\section{Evolution au cours de la vie}

Les résultats des tests statistiques ne montrent aucune différence significative entre les valeurs des $\delta^{13} \mathrm{C}_{\mathrm{ap}}$ et $\delta^{13} \mathrm{C}_{\mathrm{co}}$ des os et des dents (FIG.5,TABLEAU 7). D'une façon globale, les sujets d'Amiens auraient donc consommé, pendant leur enfance et pendant les dernières années de leur vie, des ressources provenant d'un même environnement. Dans l'hypothèse d'environnements isotopiquement distincts, une mobilité concernant l'ensemble des individus du site (déplacements de famille ou de communauté entière) pourrait donc être écartée.

En ce qui concerne les valeurs de $\delta^{15} \mathrm{~N}$, les valeurs des dents, correspondant à un signal enregistré entre 6 et 14 ans, sont statistiquement plus élevées que celles des os, correspondant à un signal enregistré dans un moment proche du décès (TABLEAU 7). Toutefois, la différence entre les valeurs de $\delta^{15} \mathrm{~N}_{\mathrm{dt}}$ et $\delta^{15} \mathrm{~N}_{\mathrm{os}}\left(\Delta^{15} \mathrm{~N}_{\mathrm{dt}-\mathrm{os}}\right)$ est très variable selon les individus (entre $-1,8$ et $+2,7 \%,+0,4 \pm 1,1 \%, \mathrm{n}=33$ ). Cette différence pourrait être le reflet d'épisodes de stress physiologique subis par la majorité des individus pendant l'enfance [63]. Il est d'ailleurs intéressant de noter que l'étude anthropologique a mis en évidence des signes osseux de stress (ligne de Harris, cribra femoris, cribra orbitalis, cribra 
mandibularis...[2]) et que les sujets présentant les valeurs de $\Delta^{15} \mathrm{~N}_{\mathrm{dt} \text {-os }}$ les plus élevées sont également ceux présentant le plus de signes osseux de stress.

Si l'on reprend les sujets particuliers identifiés comme tels par leurs valeurs isotopiques au niveau de l'os, les sujets 2104 et 2023 présentent des signatures isotopiques au niveau des tissus dentaires, qu'il s'agisse de l'email ou de la dentine, identiques à celles enregistrées au niveau des tissus osseux (sujet $2104: \delta^{13} \mathrm{C}_{\mathrm{co} / \mathrm{os}}-20,4 \%$ \% $/ \delta^{13} \mathrm{C}_{\mathrm{co} / \mathrm{dt}}-20,2 \%$; $\delta^{15} \mathrm{~N}_{\mathrm{os}} 10,0 \% / \delta^{15} \mathrm{~N}_{\mathrm{dt}} 10,5 \%$ et $\delta^{13} \mathrm{C}_{\mathrm{ap} / \mathrm{ss}}-14,9 \% / \delta^{13} \mathrm{C}_{\mathrm{ap} / \mathrm{dt}}-14,8 \%$; sujet $2023: \delta^{13} \mathrm{C}_{\mathrm{co} / \mathrm{s}}$ $-19,7 \%$ \% $/ \delta^{13} \mathrm{C}_{\mathrm{co} / \mathrm{dt}} \quad--19,8 \%$ \% $\quad \delta^{15} \mathrm{~N}_{\mathrm{os}} \quad 12,3 \%$ o $/ \delta^{15} \mathrm{~N}_{\mathrm{dt}} 12,9 \%$ et $\quad \delta^{13} \mathrm{C}_{\mathrm{ap} / \mathrm{os}}$ $-12,3 \% / \delta^{13} \mathrm{C}_{\mathrm{ap} / \mathrm{dt}}-14,5 \%$ ). Aucune variation isotopique de leur alimentation au cours de la vie n'est donc documentée. De même, le sujet 2172 présente des valeurs de $\delta^{13} \mathrm{C}$ équivalentes pour l'os comme pour la dent $\left(\delta^{13} \mathrm{C}_{\mathrm{co} / \mathrm{dt}}-15,1 \% / \delta^{13} \mathrm{C}_{\mathrm{co} / \mathrm{os}}-15,3 \% ; ; \delta^{13} \mathrm{C}_{\mathrm{ap} / \mathrm{dt}}-10,9 \% / \delta^{13} \mathrm{C}_{\mathrm{ap} / \mathrm{s}}\right.$ $-11,3 \%$ ). Les ressources alimentaires de ce sujet proviennent d'un environnement isotopiquement similaire pendant l'enfance et l'âge adulte. La différence entre la valeur de $\delta^{15} \mathrm{~N}_{\mathrm{dt}}(10,9 \%)$ et celle de $\delta^{15} \mathrm{~N}_{\mathrm{os}}(12,3 \%)$ surprend et fait encore de ce sujet un cas à part dans le site. En plus d'un âge au décès relativement jeune (21 ans), ce sujet présente des ostéochondronécroses bilatérales au niveau de l'extrémité distale de l'humérus qui seraient liées soit à une activité utilisant les membres supérieurs de manière répétitive impactant les surfaces des trochlées de manière identique, les avant-bras en extension, soit à la manifestation d'un caractère génétiquement transmissible [2].

En revanche, la valeur de $\delta^{13} \mathrm{C}_{\mathrm{co} / \mathrm{dt}}(-19,5 \%)$ du sujet 2113 est plus basse que sa valeur de $\delta^{13} \mathrm{C}_{\mathrm{co} / \mathrm{os}}\left(-18,7 \%\right.$ ) alors que ses valeurs de $\delta^{13} \mathrm{C}_{\mathrm{ap}}$ et $\delta^{15} \mathrm{~N}$ sont équivalentes quel que soit le tissu analysé. Aucune affection physiologique connue à ce jour ne modifie l'intégration du ${ }^{13} \mathrm{C}$ dans le corps [25]. Le sujet a donc, à l'âge adulte, une alimentation protéinique plus riche en ${ }^{13} \mathrm{C}$ mais une alimentation globale identique. L'alimentation du sujet à l'âge adulte comporte donc une ressource protéinique enrichie en ${ }^{13} \mathrm{C}$. La consommation de ressources marines associées de faible niveau trophique, comme les coquillages ou crustacés pourrait expliquer de telles valeurs isotopiques [4, 27].

\section{CONCLUSION}

L'objectif de cette étude visait (1) la reconstitution de l'alimentation de 33 sujets du site de la nécropole de l'Ilot de la Boucherie à Amiens (III-Vème siècles ap JC) à partir de l'analyse des isotopes stables du carbone et de l'azote d'échantillons d'apatites et de collagènes osseux et (2) l'étude des modifications alimentaires au cours de la vie des 
individus à partir d'une stratégie d'échantillonnage intra-individuelle impliquant l'analyse conjointe de tissus osseux et dentaires. Tandis que les valeurs de $\delta^{13} \mathrm{C}$ permettent la mise en évidence d'une certaine homogénéité au sein de la série en ce qui concerne le type d'environnement dans lequel les ressources alimentaires ont été puisées, la variabilité des compositions isotopiques de l'azote montre un accès varié aux protéines animales sans qu'il soit possible d'identifier le paramètre biologique ou archéologique à l'origine de cette diversité. La relation trophique observée à Amiens entre la faune et les humains échantillonnés diffère des données préalablement publiées pour des populations tardoantiques. En effet, elle serait le témoin d'une consommation non-négligeable d'une ressource enrichie en ${ }^{13} \mathrm{C}$.

L'hypothèse la plus probable semble être la consommation de millet et/ou de ressources marines associée à un apport faible en protéines animales, (viande mais aussi œufs, lait et fromage). Les données historiques laissent à penser que le millet tout comme les ressources marines possèdent un statut bien particulier pendant la période romaine. Le millet est réservé au bétail (eg [29]) tandis que les ressources marines ont un statut plus équivoque. Le schéma alimentaire visible à Amiens est peut-être à rapprocher des caractères morphologiques de cette population («germaniques») et de l'hypothèse d'une nature exogène des sujets de l'Ilot de la Boucherie [2]. L'analyse de l'évolution du signal isotopique au cours de la vie $\left(\delta^{13} \mathrm{C}_{\mathrm{ap}}\right.$ et co/os et $\delta^{15} \mathrm{~N}_{\mathrm{os}}$ versus $\delta^{13} \mathrm{C}_{\mathrm{ap}}$ et co/dt et $\left.\delta^{15} \mathrm{~N}_{\mathrm{dt}}\right)$ et sa comparaison avec celui de la faune plaide cependant pour la consommation de ressources issues de l'environnement local d'Amiens et ce, autant pendant l'enfance qu'à l'âge adulte. Si les soldats, comme les hommes restés libres, sont officiellement considérés comme citoyens, ils font tout de même l'objet d'une certaine discrimination [6] qui pourrait expliquer la persistance d'habitudes alimentaires «traditionnelles» chez les seconde et troisième générations. La population échantillonnée serait donc le reflet de ces mouvements de population prenant place au cours des III ${ }^{\text {ème }}$, IV $^{\text {ème }}$ et $V^{\text {ème }}$ siècles dans le Nord de la Gaule.

Pour quatre individus $(2104,2172,2113,2023)$ qui présentent des teneurs isotopiques de l'apatite comme du collagène sortant de la variabilité intra-site, l'hypothèse d'une migration intervenant peu de temps avant le décès est possible. Seule l'étude des compositions isotopiques du strontium et de l'oxygène pourrait permettre de conclure sur le lieu d'origine de ces sujets. De plus, afin de mieux comprendre le caractère particulier du régime alimentaire des individus concernés par la présente étude, l'analyse de l'ensemble des sujets adultes de l'Ilot de la Boucherie est en cours ainsi que celle d'individus issus d'autres nécropoles amiénoises et du Nord de la Gaule. Cette étude permettra à terme de tester le lien 
entre les caractéristiques «exogènes » de la population inhumée à l'Ilot de la Boucherie et leur alimentation.

\section{REMERCIEMENTS}

Les auteurs souhaitent remercier les éditeurs de la revue et les deux relecteurs anonymes pour leurs remarques pertinentes et leurs conseils. Nous remercions Steve Brookes d'IsoAnalytical Ltd, Crew, UK pour la réalisation des analyses spectrométriques. Nous tenons également à remercier Gwenaëlle Goude pour ses conseils relatifs à la préparation des échantillons de bioapatite et Antigone Uzunidis pour la détermination spécifique des os de faune. 
Liens d'intérêts : les auteurs déclarent ne pas avoir de lien $\delta^{13} \mathrm{C}(\% \mathrm{VPDB})$ $\delta^{15} \mathrm{~N}(\% \mathrm{AIR})$

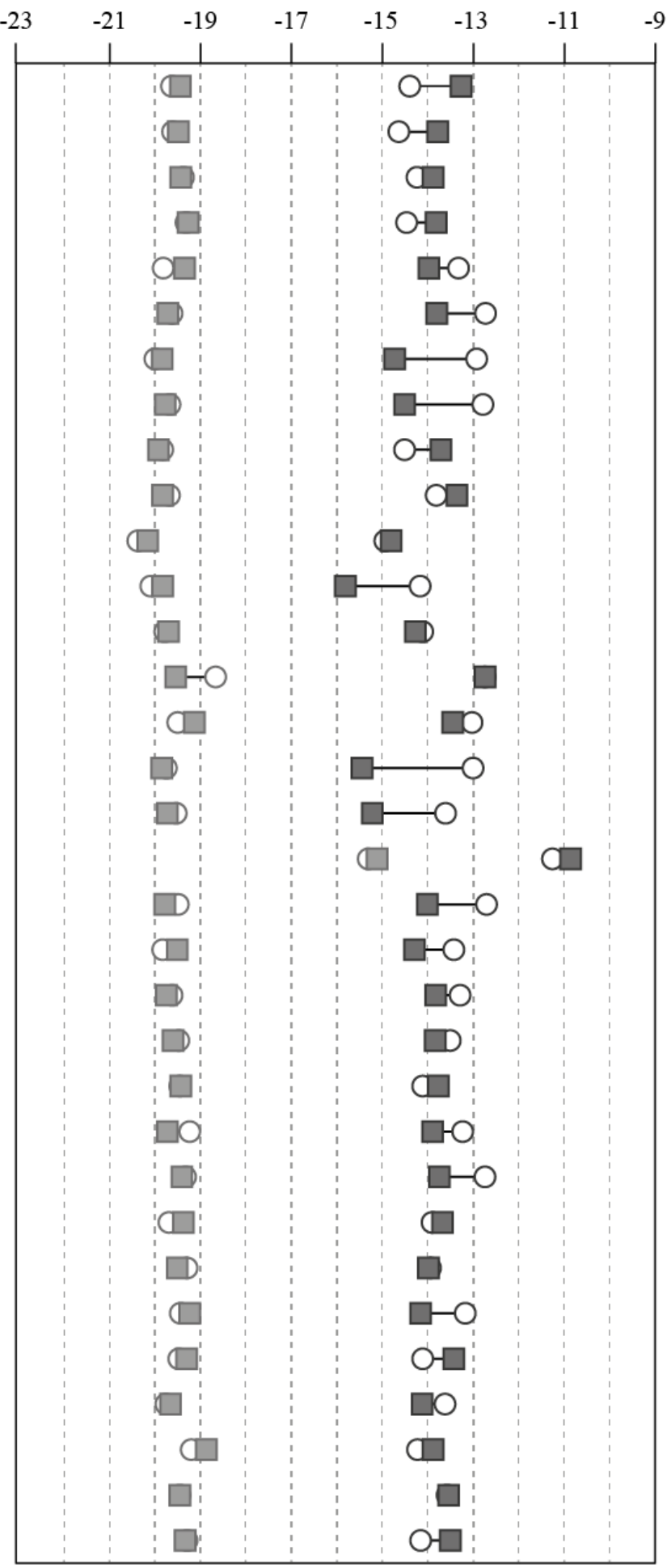

$\square$ Echantillons apatite dents $\square$ Echantillons collagène dents $\bigcirc$ Echantillons apatite os

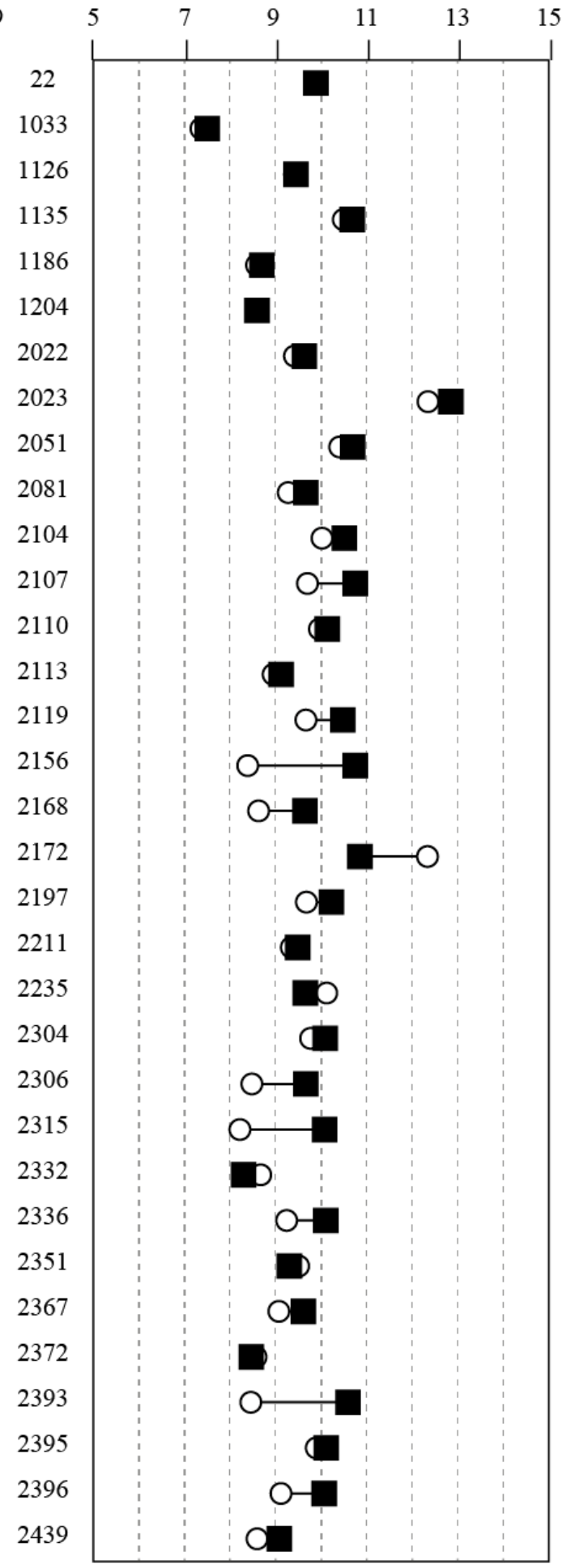

Echantillons dents $\bigcirc$ Echantillons os

d'intérêt.

\section{REFERENCES BIBLIOGRAPHIQUES}


1. Ambrose SH (1993) Isotopic analysis of paleodiets: methodological and interpretative considerations. In: Sanford MK (ed) Investigation of ancient human tissue: chemical analyze in anthropology, Gordon and Breach Science Publishers, Langhorne, pp 59-130

2. Blondiaux J (2012) Rapport anthropologique et paléopathologique. In: Binet E (ed) Amiens Îlot de la Boucherie-Rapport final d'opération. INRAP, Amiens, pp 1514-1615

3. Corbier M (2008) La fève et la murène. Hiérarchies sociales des nourritures à Rome. In: Flandrin JL, Montanari M (eds) Histoire de l'alimentation, Fayard, Paris, pp 215-3

4. Keenleyside A, Schwarcz H, Stirling L, et al (2009) Stable isotopic evidence for diet in a Roman and Late Roman population from Leptiminus, Tunisia. J Archaeol Sci 36(1):51-63

5. Prowse TL, Schwarcz HP, Saunders SR, et al (2005) Isotopic evidence for age-related variation in diet from Isola Sacra, Italy. Am J Phys Anthropol 128(1):2-13

6. Ferdiere A (2006) L'époque romaine. In: Ferdière A, Malrain F, Matterne V et al (eds) Histoire de l'agriculture en Gaule 500 avt JC-1000 ap JC, Errance, Paris, pp 65-132

7. Malrain F, Matterne V, Méniel P (2006) Le second Âge du Fer (La Tène). In: Ferdière A, Malrain F, Matterne V et al (eds) Histoire de l'agriculture en Gaule 500 avt JC-1000 ap JC, Errance, Paris, pp 11-64

8. Lepetz S, Matterne V (2003) Elevage et agriculture dans le Nord de la Gaule durant l'époque gallo-romaine: Une confrontation des données archéozoologiques et carpologiques. Rev Archéol Picardie 1(1-2):23-35

9. Lepetz S (2010) Pratiques alimentaires dans un quartier d'Amiens au $\mathrm{I}^{\text {er }}$ siècle-Les restes osseux animaux du "Palais des Sports". Rev Archéol Picardie (27):409-22

10. Lepetz S, Matterne V, Ruas MP, et al (2002) Culture et élevage en France septentrionale de l'Âge du Fer à l'an Mil. Approche carpologique et archéozoologique. In: Belmont A (ed) Autour d'Olivier De Serre - Pratiques agricoles et pensée agronomique du Néolithique aux enjeux actuels. AHSR, Rennes, pp 77-108

11. Massy JL, Bayard D (1983) Amiens romain : Samarobriva Ambianorum. Rev Archéol Picardie 2, $374 \mathrm{p}$

12. Richards MP, Hedges REM, Molleson TI, et al (1998) Stable isotope analysis reveals variations in human diet at the Poundbury Camp cemetery site. J Archaeol Sci 25(12):1247-52

13. Fuller BT, Molleson TI, Harris DA, et al (2006) Isotopic evidence for breastfeeding and possible adult dietary differences from late/sub-Roman Britain. Am J Phys Anthropol 129(1):45-54 
14. Nehlich O, Fuller BT, Jay M, et al (2011) Application of sulphur isotope ratios to examine weaning patterns and freshwater fish consumption in Roman Oxfordshire, UK. Geochim Cosmochim Acta 75(17):4963-77

15. Müldner G, Richards MP (2007) Stable isotope evidence for 1500 years of human diet at the city of York, UK. Am J Phys Anthropol 133(1):682-97

16. Müldner G, Chenery C, Eckardt H (2011) The 'Headless Romans': Multi-isotope investigations of an unusual burial ground from Roman Britain. J Archaeol Sci 38(2):28090

17. Lightfoot E, O'Connell TC, Stevens RE, et al (2009) An investigation into diet at the site of Yarnton, Oxfordshire using stable carbon and nitrogen isotopes. Oxford J Archaeol 28(3):301-22

18. Redfern RC, Hamlin C, Athfield NB (2010) Temporal changes in diet: A stable isotope analysis of late Iron Age and Roman Dorset, Britain. J Archaeol Sci 37(6):1149-60

19. Redfern RC, Millard AR, Hamlin C (2012) A regional investigation of subadult dietary patterns and health in late Iron Age and Roman Dorset, England. J Archaeol Sci 39(5):1249-59

20. Chenery C, Müldner G, Evans J, et al (2010) Strontium and stable isotope evidence for diet and mobility in Roman Gloucester, UK. J Archaeol Sci 37(1):150-63

21. Chenery C, Eckardt H, Müldner G (2011) Cosmopolitan Catterick? Isotopic evidence for population mobility on Rome's northern frontier. J Archaeol Sci 38(7):1525-36

22. Cheung C, Schroeder H, Hedges RE (2012) Diet social differentiation and cultural change in Roman Britain: new isotopic evidence from Gloucestershire. Archaeol Anthropol Sci $4(1): 61-73$

23. Fuller BT, Márquez-Grant N, Richards MP (2010) Investigation of diachronic dietary patterns on the islands of Ibiza and Formentera Spain: Evidence from carbon and nitrogen stable isotope ratio analysis. Am J Phys Anthropol 143(4):512-22

24. Nehlich O, Fuller BT, Márquez-Grant N, et al (2012) Investigation of diachronic dietary patterns on the islands of Ibiza and Formentera Spain: Evidence from sulfur stable isotope ratio Analysis. Am J Phys Anthropol 149(1):115-24

25. Prowse TL, Schwarcz HP, Saunders SR, et al (2004) Isotopic paleodiet studies of skeletons from the Imperial Roman-age cemetery of Isola Sacra, Rome, Italy. J Archaeol Sci 31(3):258-72 
26. Prowse TL, Schwarcz HP, Saunders SR, et al (2008) Isotopic and dental evidence for weaning and young child feeding practices in an Imperial Roman skeletal sample. Am J Phys Anthropol 137(3):294-308

27. Craig OE, Biazzo M, O'connell TC, et al (2009) Stable isotopic evidence for diet at the Imperial Roman coastal site of Velia (1st and 2nd Centuries AD) in southern Italy. Am J Phys Anthropol 139(4):572-83

28. Rutgers LV, Van Strydonck M, Boudin M, et al (2009) Stable isotope data from the Early Christian Catacombs of ancient Rome: New insights into the dietary habits of Rome's Early Christians. J Archaeol Sci 36(5):1127-34

29. Killgrove K, Tykot RH (2013) Food for Rome: A stable isotope investigation of diet in the Imperial period (1st-3rd centuries AD). J Anthropol Archaeol 32(1):28-38

30. Hakenbeck S, McManus E, Geisler H, et al (2010) Diet and mobility in Early Medieval Bavaria: A study of carbon and nitrogen stable isotopes. Am J Phys Anthropol 143(2):235-49

31. Reitsema LJ, Kozłowski T (2013) Diet in Poland before the state: Stable isotope evidence from a Wielbark population (2nd c. AD). Anthropol Rev 76(1):1-22

32. Lösch S, Moghaddam N, Grossschmidt K, et al (2014) Stable isotope and trace element studies on gladiators and contemporary romans from Ephesus (Turkey, 2nd and 3rd Ct. AD): Implications for differences in diet. PLoS ONE 9(10):e110489

33. Lightfoot E, Šlaus M, O'connell TC (2012) Changing cultures, changing cuisines: cultural transitions and dietary change in Iron Age, Roman and Early Medieval Croatia. Am J Phys Anthropol 148(4):543-56

34. Delaplace C, France J (2005) Histoire des Gaules VIe s. av. J.C. - VIe s. ap. J.-C., 3ème éd., Armand Colin, Paris, 233 p

35. Ferdière A (2005) Les Gaules, Ie s. av. J.-C. - Ve s. ap. J.-C. Armand Colin, Paris 448 p

36. Bruzek J (2002) A method for visual determination of sex using the human hip bone. Am J Phys Anthropol 117(2):157-68

37. Buikstra JE, Ubelaker D (1994) Standards for data collection from human skeletal remains: proceedings of a seminar at the Field Museum of Natural History. Arkansas Archeological Survey, Fayetteville, $224 \mathrm{p}$

38. Harris EF, Buck AL (2002) Tooth mineralization: a technical note on the MoorreesFanning-Hunt standards. Dent Anthropol 16:15-20

39. Moorrees CFA, Fanning EA, Hunt EE (1963) Age variation of formation stages for ten permanent teeth. J Dental Research 42(6):1490-1502 
40. Hedges REM, Clement JG, Thomas CD, et al (2007) Collagen turnover in the adult femoral mid-shaft: modeled from anthropogenic radiocarbon tracer measurements. Am J Phys Anthropol 133(2):808-16

41. Minagawa M, Wada E (1984) Stepwise enrichment of ${ }^{15} \mathrm{~N}$ along food chains: further evidence and the relation between $\delta^{15} \mathrm{~N}$ and animal age. Geochim Cosmochim Acta 48(5):1135-40

42. Bocherens H, Drucker DG (2003) Trophic level isotopic enrichment of carbon and nitrogen in bone collagen: case studies from recent and ancient terrestrial ecosystems. Intern J Osteoarchaeol 13:46-53

43. Kellner CM, Schoeninger MJ (2007) A simple carbon isotope model for reconstructing prehistoric human diet. Am J Phys Anthropol 133(4):1112-27

44. Froehle AW, Kellner CM, Schoeninger MJ (2010) Focus: Effect of diet and protein source on carbon stable isotope ratios in collagen: follow up to Warinner and Tuross (2009). J Archaeol Sci 37(10):2662-701

45. Deines P (1980) The isotopic composition of reduced organic carbon. In: Fritz P, Fontes JC (eds) Handbook of environmental isotope geochemistry, Elsevier, New-York, pp 329406

46. Schoeninger MJ, DeNiro MJ (1984) Nitrogen and carbon isotopic composition of bone collagen from marine and terrestrial animals. Geochim Cosmochim Acta 48:625-39

47. Farquhar GD, Ehleringer JR, Hubick KT (1989) Carbon isotope discrimination and photosynthesis. Annu Rev Plant Biol 40(1):503-37

48. Hillson S (2005) Teeth. $2^{\text {nd }}$ Edition. Cambridge University Press, New York, $373 \mathrm{p}$

49. Longin R (1971) New method of collagen extraction for radiocarbon dating. Nature 230:241-2

50. Bocherens H, Fizet M, Mariotti A, et al (1991) Isotopic biogeochemistry $\left({ }^{13} \mathrm{C},{ }^{15} \mathrm{~N}\right)$ of fossil vertebrate collagen: application to the study of a past food web including Neandertal Man. J Hum Evol 20(6):481-92

51. Balasse M, Ambrose SH, Smith AB, et al (2002) The seasonal mobility model for prehistoric herders in the south-western Cape of South Africa assessed by isotopic analysis of sheep tooth enamel. J Archaeol Sci 29(9):917-32

52. Ambrose SH (1990) Preparation and characterization of bone and tooth collagen for isotopic analysis. J Archaeol Sci 17(4):431-51

53. DeNiro MJ (1985) Post-Mortem preservation and alteration of in vivo bone collagen isotope ratios in relation to paleodietary reconstruction. Nature 317:806-9 
54. Van Klinken GJ (1999) Bone collagen quality indicators for palaeodietary and radiocarbon measurements. J Archaeol Sci 26(6):687-95

55. Zazzo A (2014) Bone and enamel carbonate diagenesis: a radiocarbon prospective. Palaeogeogr Palaeoclimatol Palaeoecol 416:168-78

56. Salesse K, Dufour E, Lebon M, et al (2014) Variability of bone preservation in a confined environment: the case of the catacomb of Sts Peter and Marcellinus (Rome, Italy). Palaeogeogr Palaeoclimatol Palaeoecol 416:43-54

57. Eerkens JW, Mackie M, Bartelink EJ (2013) Brackish water foraging: Isotopic landscapes and dietary reconstruction in Suisun Marsh, Central California. J Archaeol Sci 40(8):3270-81

58. Froehle AW, Kellner CM, Schoeninger MJ (2012) Multivariate carbon and nitrogen stable isotope model for the reconstruction of prehistoric human diet. Am J Phys Anthropol 37(10):2662-70

59. Bocherens H (1999) Isotopes stables et reconstitution du régime alimentaire des hominidés fossiles: une revue. Bull Mem Soc Anthropol Paris 11(3):261-87

60. Hamilton J, Hedges REM, Robinson M (2009) Rooting for pigfruit: pig feeding in Neolithic and Iron Age Britain compared. Antiquity 83:998-1011

61. Varron (2003) De l'agriculture. Errance, Paris, 96 p

62. Dufour E, Bocherens H, Mariotti A (1999) Paleodietary implications of isotopic variability in Eurasian Lacustrine fish J Archaeol Sci 26(6):617-27

63. Fuller BT, Fuller JL, Sage NE, et al (2005) Nitrogen balance and $\delta^{15} \mathrm{~N}$ : why you're not what you eat during nutritional stress. Rapid Commun Mass Spectrom 19(18):2497-2506

64. Fuller BT, Müldner G, Van Neer W, et al (2012) Carbon and Nitrogen Stable Isotope Ratio Analysis of Freshwater, Brackish and Marine Fish from Belgian Archaeological Sites (1st and 2nd Millennium AD). J Anal At Spectrom 27(5):807-20

65. Müldner G, Richards MP (2007) Stable Isotope Evidence for 1500 Years of Human Diet at the City of York, UK. Am J Phys Anthropol 133(1):682-97 


\section{LEGENDES}

Tableau 1-Données élémentaires et isotopiques des échantillons de collagène - Elemental and isotopic data from collagen samples

Tableau 2-Données élémentaires et isotopiques des échantillons d'apatite - Elemental and isotopic data from apatite samples

Tableau 3-Données élémentaires et isotopiques du corpus de faune - Elemental and isotopic data from the faunal corpus

Tableau 4-Résultats des tests de corrélation de rang de Spearman - Spearman correlation tests results

Tableau 5-Résultats des tests approximatifs (Monte Carlo, 10000 réplicats) de Kruskal Wallis - Approximative Kruskal Wallis test results (Monte Carlo, 10000 replicats)

Tableau 6-Résultats des tests exacts de Wilcoxon bivariés - Wilcoxon exact unpaired tests results

Tableau 7-Résultats des tests exacts de Wilcoxon appariés- Wilcoxon exact paired tests results

Tableau 8-Statistiques récapitulatives des valeurs isotopiques des groupes humains - Statistics of isotope values acccording to human groups

Figure 1-Situation géographique du site archéologique de l'Ilot de la Boucherie, Amiens, Somme, France - Geographic context of the archaeological site of Ilot de la Boucherie, Amiens, Somme, France

Figure 1-Valeurs isotopiques $\left(\delta^{13} \mathrm{C}_{\mathrm{co}} / \delta^{15} \mathrm{~N}\right.$, os $)$ des sujets d'Amiens, du corpus de faune et des ressources marines publiées pour la Manche [65,66]-Isotope values $\left(\delta^{13} \mathrm{C}_{\mathrm{co}} / \delta^{15} \mathrm{~N}\right.$, bone) from the subjects of Amiens, the faunal corpus and the published marine ressources of the Channel Sea $[65,66]$

Figure 3-Valeurs isotopiques du carbone (os) selon le modèle mono-isotopique [43,44,57] Carbon isotope values (bone) according to the simple carbon isotope model $[43,44,57]$

Figure 4-Valeurs isotopiques $\left(\delta^{13} \mathrm{C}_{\mathrm{ap}}, \delta^{13} \mathrm{C}_{\mathrm{co}}\right.$ et $\delta^{15} \mathrm{~N}$, os) selon le modèle multi -isotopique des fonctions discriminantes [56] - Isotope values $\left(\delta^{13} \mathrm{C}_{\mathrm{ap}}, \delta^{13} \mathrm{C}_{\mathrm{co}}\right.$ and $\delta^{15} \mathrm{~N}$, bone $)$ according to the multi -isotopic model of discriminant functions [56]

Figure 5-Comparaison des valeurs isotopiques $\left(\delta^{13} \mathrm{C}_{\mathrm{ap}}, \delta^{13} \mathrm{C}_{\mathrm{co}}\right.$ et $\left.\delta^{15} \mathrm{~N}\right)$ des tissus osseux et dentaires pour les 33 individus humains étudiés-Comparison of isotope values $\left(\delta^{13} \mathrm{C}_{\mathrm{ap}}, \delta^{13} \mathrm{C}_{\mathrm{co}}\right.$ and $\delta^{15} \mathrm{~N}$ ) from bone and dental tissus of the 33 human specimen studied 
Tableau 1-Données élémentaires et isotopiques des échantillons de collagène Elemental and isotopic data from collagen samples

\begin{tabular}{|c|c|c|c|c|c|c|c|c|c|c|}
\hline Sép. & Sexe & Age* & Sect. & Elément & $\begin{array}{c}\text { Rendement } \\
\left(\mathrm{mg}^{\left.-\mathrm{g}^{-1}\right)}\right.\end{array}$ & $\% \mathrm{C}$ & $\% \mathrm{~N}$ & $\mathrm{C} / \mathrm{N}$ & $\delta^{13} \mathrm{C}_{\mathrm{co}}(\% \mathrm{o})$ & $\delta^{15} \mathrm{~N}(\%)$ \\
\hline \multirow[t]{2}{*}{22} & $\mathrm{H}$ & 41 & 1 & $\mathrm{P} 1$ & 166,7 & 38,2 & 13,9 & 3,2 & $-19,4$ & 9,9 \\
\hline & & & & PPM & 99,1 & 42,7 & 15,6 & 3,2 & $-19,6$ & 9,9 \\
\hline \multirow[t]{2}{*}{1033} & $\mathrm{~F}$ & 32 & HS & $\mathrm{P} 1$ & 122,0 & 38,5 & 14,1 & 3,2 & $-19,5$ & 7,5 \\
\hline & & & & MTT4d & 113,6 & 40,9 & 14,9 & 3,2 & $-19,6$ & 7,4 \\
\hline \multirow[t]{2}{*}{1126} & $\mathrm{H}$ & 29 & 2 & P1 & 136,7 & 38,3 & 13,9 & 3,2 & $-19,4$ & 9,4 \\
\hline & & & & Côte & 44,8 & 38,3 & 14,0 & 3,2 & $-19,4$ & 9,4 \\
\hline \multirow{2}{*}{1135} & $\mathrm{~F}$ & 56 & 2 & $\mathrm{P} 2$ & 129,9 & 36,2 & 13,1 & 3,2 & $-19,3$ & 10,7 \\
\hline & & & & PPM & 163,2 & 38,7 & 14,0 & 3,2 & $-19,3$ & 10,5 \\
\hline \multirow[t]{2}{*}{1186} & $\mathrm{H}$ & 38 & 3 & P2 & 131,2 & 38,0 & 13,9 & 3,2 & $-19,3$ & 8,7 \\
\hline & & & & PPM & 147,4 & 37,4 & 13,6 & 3,2 & $-19,8$ & 8,6 \\
\hline \multirow[t]{2}{*}{1204} & $\mathrm{~F}$ & 61 & 3 & $\mathrm{P} 1$ & 108,8 & 33,3 & 12,0 & 3,2 & $-19,7$ & 8,6 \\
\hline & & & & Côte & 46,3 & 38,6 & 14,0 & 3,2 & $-19,6$ & 8,6 \\
\hline \multirow[t]{2}{*}{2022} & $\mathrm{H}$ & 21 & 4 & P1 & 141,6 & 34,2 & 12,5 & 3,2 & $-19,8$ & 9,6 \\
\hline & & & & PPM & 52,3 & 39,8 & 14,5 & 3,2 & $-20,0$ & 9,4 \\
\hline \multirow{2}{*}{2023} & $\mathrm{H}$ & 20 & 4 & $\mathrm{P} 1$ & 173,1 & 38,6 & 14,0 & 3,2 & $-19,8$ & 12,9 \\
\hline & & & & PPM & 54,3 & 41,0 & 15,1 & 3,2 & $-19,7$ & 12,3 \\
\hline \multirow[t]{2}{*}{2051} & F & 20 & 4 & $\mathrm{P} 1$ & 119,3 & 37,2 & 13,2 & 3,3 & $-19,9$ & 10,7 \\
\hline & & & & PPM & 152,8 & 41,7 & 15,2 & 3,2 & $-19,8$ & 10,4 \\
\hline \multirow[t]{2}{*}{2081} & $\mathrm{H}$ & 27 & 4 & $\mathrm{P} 2$ & 156,3 & 39,4 & 14,1 & 3,3 & $-19,8$ & 9,7 \\
\hline & & & & PPM & 183,4 & 41,8 & 15,2 & 3,2 & $-19,7$ & 9,3 \\
\hline \multirow[t]{2}{*}{2104} & $\mathrm{H}$ & 46 & 5 & P2 & 137,5 & 41,0 & 14,9 & 3,2 & $-20,2$ & 10,5 \\
\hline & & & & PPM & 175,6 & 39,1 & 14,3 & 3,2 & $-20,4$ & 10,0 \\
\hline \multirow{2}{*}{2107} & $\mathrm{H}$ & 25 & 4 & P1 & 130,3 & 37,6 & 13,9 & 3,2 & $-19,8$ & 10,7 \\
\hline & & & & PPM & 172,3 & 40,1 & 14,7 & 3,2 & $-20,1$ & 9,7 \\
\hline \multirow[t]{2}{*}{2110} & F & 37 & 4 & $\mathrm{P} 2$ & 173,1 & 37,1 & 13,5 & 3,2 & $-19,7$ & 10,1 \\
\hline & & & & PMM & 194,4 & 41,0 & 14,9 & 3,2 & $-19,8$ & 10,0 \\
\hline \multirow[t]{2}{*}{2113} & F & 24 & 4 & P1 & 165,2 & 41,3 & 15,0 & 3,2 & $-19,5$ & 9,1 \\
\hline & & & & PPM & 176,5 & 40,7 & 14,9 & 3,2 & $-18,7$ & 8,9 \\
\hline \multirow[t]{2}{*}{2119} & Ind & 15 & 4 & P1 & 156,4 & 39,3 & 14,4 & 3,2 & $-19,1$ & 10,5 \\
\hline & & & & Côte & 89,2 & 39,5 & 14,5 & 3,2 & $-19,5$ & 9,7 \\
\hline \multirow[t]{2}{*}{2156} & $\mathrm{H}$ & 36 & 5 & $\mathrm{P} 2$ & 115,6 & 36,8 & 13,4 & 3,2 & $-19,9$ & 10,7 \\
\hline & & & & PPM & 41,8 & 35,5 & 13,4 & 3,1 & $-19,8$ & 8,4 \\
\hline 2168 & $\mathrm{~F}$ & 14 & 5 & $\mathrm{P} 1$ & 55,0 & 39,7 & 14,3 & 3,2 & $-19,7$ & 9,6 \\
\hline & & & & Côte & 105,5 & 40,9 & 15,0 & 3,2 & $-19,5$ & 8,6 \\
\hline 2172 & F & 21 & 5 & $\mathrm{P} 1$ & 180,1 & 39,8 & 14,6 & 3,2 & $-15,1$ & 10,9 \\
\hline & & & & Côte & 179,0 & 44,2 & 16,0 & 3,2 & $-15,3$ & 12,3 \\
\hline 2197 & $\mathrm{~F}$ & 59 & 6 & P2 & 146,0 & 38,3 & 13,9 & 3,2 & $-19,8$ & 10,2 \\
\hline & & & & PPP & 133,0 & 31,2 & 11,3 & 3,2 & $-19,5$ & 9,7 \\
\hline 2211 & $\mathrm{~F}$ & 14,5 & 3 & $\mathrm{P} 1$ & 127,9 & 36,2 & 13,0 & 3,2 & $-19,5$ & 9,5 \\
\hline & & & & Côte & 35,6 & 31,9 & 11,7 & 3,2 & $-19,8$ & 9,3 \\
\hline 2235 & $\mathrm{~F}$ & 34 & 6 & $\mathrm{C}$ & 141,9 & 37,9 & 13,8 & 3,2 & $-19,8$ & 9,6 \\
\hline & & & & PPM & 29,1 & 36,5 & 13,2 & 3,2 & $-19,6$ & 10,1 \\
\hline 2304 & $\mathrm{~F}$ & 25 & 5 & $\mathrm{P} 2$ & 187,4 & 40,2 & 14,6 & 3,2 & $-19,6$ & 10,1 \\
\hline & & & & PPM & 164,8 & 40,3 & 14,6 & 3,2 & $-19,5$ & 9,8 \\
\hline 2306 & $\mathrm{~F}$ & 47 & 5 & M1 & 149,4 & 39,1 & 14,1 & 3,2 & $-19,4$ & 9,7 \\
\hline & & & & PMM & 162,0 & 40,3 & 14,7 & 3,2 & $-19,5$ & 8,5 \\
\hline 2315 & $\mathrm{H}$ & 38 & 5 & $\mathrm{P} 2$ & 82,0 & 37,0 & 13,2 & 3,3 & $-19,7$ & 10,1 \\
\hline & & & & Côte & 92,6 & 36,5 & 14,1 & 3,0 & $-19,2$ & 8,2 \\
\hline 2332 & $\mathrm{H}$ & 48 & 4 & $\mathrm{P} 2$ & 155,7 & 40,5 & 14,6 & 3,2 & $-19,4$ & 8,3 \\
\hline & & & & Côte & 79,8 & 37,6 & 13,7 & 3,2 & $-19,3$ & 8,7 \\
\hline 2336 & $\mathrm{H}$ & 41 & 5 & $\mathrm{P} 1$ & 186,2 & 39,8 & 14,5 & 3,2 & $-19,4$ & 10,1 \\
\hline & & & & PPM & 139,6 & 42,5 & 15,9 & 3,1 & $-19,7$ & 9,2 \\
\hline 2351 & $\mathrm{H}$ & 63 & 5 & P2 & 197,6 & 41,5 & 15,1 & 3,2 & $-19,5$ & 9,3 \\
\hline & & & & MTT2g & 151,0 & 40,7 & 14,7 & 3,2 & $-19,3$ & 9,5 \\
\hline 2367 & $\mathrm{H}$ & 56 & 5 & $\mathrm{P} 2$ & 195,3 & 41,6 & 15,1 & 3,2 & $-19,2$ & 9,6 \\
\hline
\end{tabular}




\begin{tabular}{|c|c|c|c|c|c|c|c|c|c|c|}
\hline Sép. & Sexe & Age* & Sect. & Elément & $\begin{array}{c}\text { Rendement } \\
\left(\mathrm{mg}^{-\mathrm{g}^{-1}}\right)\end{array}$ & $\% \mathrm{C}$ & $\% \mathrm{~N}$ & $\mathrm{C} / \mathrm{N}$ & $\delta^{13} \mathbf{C}_{\mathrm{co}}(\% \mathrm{o})$ & $\delta^{15} \mathbf{N}(\% 0)$ \\
\hline \multirow{3}{*}{2372} & & & & PPM & 103,2 & 42,5 & 15,6 & 3,2 & $-19,4$ & 9,1 \\
\hline & $\mathrm{F}$ & 55 & 5 & M1 & 161,9 & 40,8 & 14,8 & 3,2 & $-19,3$ & 8,5 \\
\hline & & & & PPP & 86,2 & 39,3 & 14,6 & 3,1 & $-19,5$ & 8,6 \\
\hline \multirow[t]{2}{*}{2393} & $\mathrm{~F}$ & 40 & 5 & $\mathrm{P} 1$ & 191,1 & 39,0 & 14,2 & 3,2 & $-19,7$ & 10,6 \\
\hline & & & & MTT3g & 171,4 & 42,0 & 15,2 & 3,2 & $-19,8$ & 8,5 \\
\hline \multirow[t]{2}{*}{2395} & $\mathrm{H}$ & 51 & 5 & $\mathrm{P} 1$ & 180,6 & 37,9 & 13,6 & 3,3 & $-18,9$ & 10,1 \\
\hline & & & & PPM & 153,0 & 41,5 & 15,0 & 3,2 & $-19,2$ & 9,9 \\
\hline \multirow[t]{2}{*}{2396} & $\mathrm{~F}$ & 18 & 5 & $\mathrm{P} 1$ & 111,1 & 36,8 & 13,4 & 3,2 & $-19,5$ & 10,1 \\
\hline & & & & PPMd & 167,4 & 39,1 & 14,3 & 3,2 & $-19,5$ & 9,1 \\
\hline \multirow[t]{12}{*}{2439} & $\mathrm{~F}$ & 54 & 5 & $\mathrm{C}$ & 151,3 & 39,7 & 14,3 & 3,2 & $-19,3$ & 9,1 \\
\hline & & & & Côte & 138,8 & 39,0 & 14,2 & 3,2 & $-19,3$ & 8,6 \\
\hline & & & Dents & Maximum & 197,6 & 41,6 & 15,1 & 3,3 & $-15,1$ & 12,9 \\
\hline & & & & Minimum & 55,0 & 33,3 & 12,0 & 3,2 & $-20,2$ & 7,5 \\
\hline & & & & Moyenne & 147,4 & 38,5 & 14,0 & 3,2 & $-19,4$ & 9,8 \\
\hline & & & & Ecart-type & 32,6 & 2,0 & 0,7 & 0,0 & 0,8 & 1,0 \\
\hline & & & & Médiane & 149,4 & 38,5 & 14,0 & 3,2 & $-19,5$ & 9,9 \\
\hline & & & Os & Maximum & 194,4 & 44,2 & 16,0 & 3,2 & $-15,3$ & 12,3 \\
\hline & & & & Minimum & 29,1 & 31,2 & 11,3 & 3,0 & $-20,4$ & 7,4 \\
\hline & & & & Moyenne & 121,2 & 39,5 & 14,4 & 3,2 & $-19,4$ & 9,4 \\
\hline & & & & Ecart-type & 51,3 & 2,8 & 1,0 & 0,0 & 0,8 & 1,0 \\
\hline & & & & Médiane & 138,8 & 40,1 & 14,6 & 3,2 & $-19,5$ & 9,3 \\
\hline
\end{tabular}

*les âges sont donnés en années, l'imprécision des âges individuels est de l'ordre de 4 ans, $\mathrm{F}$ : femme, H : homme, Ind : indeterminé, HS : Hors secteur, C : canine ; P1 : première prémolaire ; $\mathrm{P} 2$ : deuxième prémolaire ; M1 : première molaire ; PPM : phalange proximale de la main, PMM : phalange médiale de la main ; PPP : phalange proximale du pied, MTT2, MTT3 ou MTT4 : métatarse 1, 2, 3 ou 4 ; d :droite ; g : gauche. 
Tableau 2-Données élémentaires et isotopiques des échantillons d'apatite - Elemental and isotopic data from apatite samples

\begin{tabular}{|c|c|c|c|c|c|c|c|c|c|}
\hline Sép. & Sexe & Age* $^{*}$ & Sect. & Elément & Rendement (\%) & Poids (mg) & $\mathrm{Vol} \mathrm{CO}_{2}(\mathrm{ml})$ & $\% \mathrm{CO}_{3}$ & $\delta^{13} \mathrm{C}_{\mathrm{ap}}(\%$ \% $)$ \\
\hline \multirow[t]{2}{*}{22} & $\mathrm{H}$ & 41 & 1 & P1 & 57,6 & 4,3 & 0,053 & 6,1 & $-13,3$ \\
\hline & & & & PPM & 22,2 & 4,6 & 0,049 & 4,8 & $-14,4$ \\
\hline \multirow[t]{2}{*}{1033} & $\mathrm{~F}$ & 32 & HS & $\mathrm{P} 1$ & 62,4 & 4,7 & 0,043 & 4,5 & $-13,8$ \\
\hline & & & & MTT4d & 39,6 & 6,1 & 0,060 & 4,5 & $-14,6$ \\
\hline \multirow[t]{2}{*}{1126} & $\mathrm{H}$ & 29 & 2 & $\mathrm{P} 1$ & 47,1 & 2,7 & 0,027 & 4,9 & $-13,9$ \\
\hline & & & & Côte & 53,4 & 6,1 & 0,060 & 4,4 & $-14,2$ \\
\hline \multirow[t]{2}{*}{1135} & $\mathrm{~F}$ & 56 & 2 & $\mathrm{P} 2$ & 53,6 & 4,6 & 0,049 & 5,1 & $-13,8$ \\
\hline & & & & PPM & 47,0 & 6,1 & 0,067 & 4,9 & $-14,5$ \\
\hline \multirow[t]{2}{*}{1186} & $\mathrm{H}$ & 38 & 3 & $\mathrm{P} 2$ & 67,4 & 5,5 & 0,057 & 4,6 & $-14,0$ \\
\hline & & & & PPM & 31,0 & 6,4 & 0,084 & 5,9 & $-13,3$ \\
\hline \multirow[t]{2}{*}{1204} & $\mathrm{~F}$ & 61 & 3 & $\mathrm{P} 1$ & 57,6 & 5,2 & 0,061 & 5,3 & $-13,8$ \\
\hline & & & & Côte & 35,5 & 5,3 & 0,095 & 8,1 & $-12,7$ \\
\hline \multirow[t]{2}{*}{2022} & $\mathrm{H}$ & 21 & 4 & $\mathrm{P} 1$ & 53,1 & 4,9 & 0,046 & 4,2 & $-14,7$ \\
\hline & & & & PPM & 26,7 & 5,4 & 0,104 & 8,7 & $-12,9$ \\
\hline \multirow[t]{2}{*}{2023} & $\mathrm{H}$ & 20 & 4 & $\mathrm{P} 1$ & 42,3 & 3,2 & 0,033 & 4,6 & $-14,5$ \\
\hline & & & & PPM & 31,3 & 6,6 & 0,128 & 8,7 & $-12,8$ \\
\hline \multirow[t]{2}{*}{2051} & $\mathrm{~F}$ & 20 & 4 & $\mathrm{P} 1$ & 60,2 & 4,7 & 0,045 & 4,3 & $-13,7$ \\
\hline & & & & PPM & 29,9 & 5,2 & 0,062 & 5,3 & $-14,5$ \\
\hline \multirow[t]{2}{*}{2081} & $\mathrm{H}$ & 27 & 4 & P2 & 66,0 & 5,8 & 0,047 & 3,6 & $-13,4$ \\
\hline & & & & PPM & 44,8 & 8,9 & 0,089 & 4,5 & $-13,8$ \\
\hline \multirow{2}{*}{2104} & $\mathrm{H}$ & 46 & 5 & $\mathrm{P} 2$ & 61,0 & 5,1 & 0,045 & 4,0 & $-14,8$ \\
\hline & & & & PPM & 31,0 & 5,6 & 0,046 & 3,7 & $-14,9$ \\
\hline \multirow[t]{2}{*}{2107} & $\mathrm{H}$ & 25 & 4 & P1 & 53,1 & 4,0 & 0,038 & 4,3 & $-15,8$ \\
\hline & & & & PPM & 43,8 & 8,3 & 0,065 & 3,5 & $-14,2$ \\
\hline \multirow[t]{2}{*}{2110} & $\mathrm{~F}$ & 37 & 4 & $\mathrm{P} 2$ & 69,8 & 5,7 & 0,053 & 4,1 & $-14,3$ \\
\hline & & & & PMM & 45,9 & 8,3 & 0,086 & 4,6 & $-14,1$ \\
\hline \multirow[t]{2}{*}{2113} & $\mathrm{~F}$ & 24 & 4 & P1 & 62,0 & 5,4 & 0,060 & 5,0 & $-12,7$ \\
\hline & & & & PPM & 44,9 & 8,3 & 0,061 & 3,3 & $-12,7$ \\
\hline \multirow{2}{*}{2119} & Ind & 15 & 4 & $\mathrm{P} 1$ & 66,3 & 5,1 & 0,051 & 4,5 & $-13,5$ \\
\hline & & & & Côte & 40,2 & 7,5 & 0,080 & 4,8 & $-13,0$ \\
\hline \multirow[t]{2}{*}{2156} & $\mathrm{H}$ & 36 & 5 & $\mathrm{P} 2$ & 44,0 & 3,9 & 0,047 & 5,4 & $-15,5$ \\
\hline & & & & PPM & 35,3 & 6,7 & 0,093 & 6,2 & $-13,0$ \\
\hline 2168 & $\mathrm{~F}$ & 14 & 5 & $\mathrm{P} 1$ & 61,9 & 5,2 & 0,042 & 3,6 & $-15,2$ \\
\hline & & & & Côte & 28,4 & 4,2 & 0,061 & 6,6 & $-13,6$ \\
\hline 2172 & $\mathrm{~F}$ & 21 & 5 & $\mathrm{P} 1$ & 59,0 & 5,1 & 0,050 & 4,5 & $-10,9$ \\
\hline & & & & Côte & 23,1 & 4,8 & 0,040 & 3,7 & $-11,3$ \\
\hline 2197 & $\mathrm{~F}$ & 59 & 6 & $\mathrm{P} 2$ & 69,0 & 6,1 & 0,057 & 4,2 & $-14,0$ \\
\hline & & & & PPP & 30,1 & 5,3 & 0,081 & 6,8 & $-12,7$ \\
\hline 2211 & $\mathrm{~F}$ & 14,5 & 3 & $\mathrm{P} 1$ & 55,7 & 4,5 & 0,045 & 4,5 & $-14,3$ \\
\hline & & & & Côte & 25,0 & 2,7 & 0,037 & 6,0 & $-13,4$ \\
\hline 2235 & $\mathrm{~F}$ & 34 & 6 & $\mathrm{C}$ & 52,1 & 4,3 & 0,054 & 5,5 & $-13,8$ \\
\hline & & & & PPM & 25,1 & 5,0 & 0,067 & 6,0 & $-13,3$ \\
\hline 2304 & $\mathrm{~F}$ & 25 & 5 & $\mathrm{P} 2$ & 61,6 & 5,3 & 0,057 & 4,9 & $-13,8$ \\
\hline & & & & PPM & 42,5 & 7,8 & 0,063 & 3,6 & $-13,5$ \\
\hline 2306 & $\mathrm{~F}$ & 47 & 5 & M1 & 46,9 & 3,4 & 0,038 & 5,0 & $-13,8$ \\
\hline & & & & PMM & 26,3 & 4,9 & 0,042 & 3,9 & $-14,1$ \\
\hline 2315 & $\mathrm{H}$ & 38 & 5 & $\mathrm{P} 2$ & 53,5 & 4,6 & 0,040 & 3,9 & $-13,9$ \\
\hline & & & & CÔTE & 40,7 & 8,1 & 0,088 & 4,9 & $-13,2$ \\
\hline 2332 & $\mathrm{H}$ & 48 & 4 & $\mathrm{P} 2$ & 44,3 & 3,8 & 0,042 & 4,8 & $-13,7$ \\
\hline & & & & Côte & 37,2 & 6,7 & 0,154 & 10,4 & $-12,7$ \\
\hline 2336 & $\mathrm{H}$ & 41 & 5 & $\mathrm{P} 1$ & 53,7 & 2,7 & 0,024 & 4,1 & $-13,7$ \\
\hline & & & & PPM & 40,1 & 7,8 & 0,052 & 2,9 & $-13,9$ \\
\hline 2351 & $\mathrm{H}$ & 63 & 5 & $\mathrm{P} 2$ & 60,6 & 5,4 & 0,055 & 4,5 & $-14,0$ \\
\hline & & & & MTT2g & 43,2 & 7,7 & 0,067 & 3,9 & $-13,9$ \\
\hline 2367 & $\mathrm{H}$ & 56 & 5 & P2 & 61,0 & 5,2 & 0,060 & 5,1 & $-14,2$ \\
\hline
\end{tabular}




\begin{tabular}{|c|c|c|c|c|c|c|c|c|c|}
\hline Sép. & Sexe & Age* & Sect. & Elément & Rendement (\%) & Poids (mg) & $\mathrm{Vol} \mathrm{CO}_{2}(\mathrm{ml})$ & $\% \mathrm{CO}_{3}$ & $\delta^{13} C_{a p}(\% o)$ \\
\hline & & & & PPM & 45,8 & 8,2 & 0,094 & 5,1 & $-13,2$ \\
\hline \multirow[t]{2}{*}{2372} & $\mathrm{~F}$ & 55 & 5 & M1 & 65,0 & 5,5 & 0,044 & 3,6 & $-13,4$ \\
\hline & & & & PPP & 30,4 & 6,0 & 0,077 & 5,8 & $-14,1$ \\
\hline \multirow[t]{2}{*}{2393} & $\mathrm{~F}$ & 40 & 5 & $\mathrm{P} 1$ & 67,7 & 5,7 & 0,049 & 3,8 & $-14,1$ \\
\hline & & & & MTT3g & 40,8 & 8,0 & 0,047 & 2,6 & $-13,6$ \\
\hline \multirow[t]{2}{*}{2395} & $\mathrm{H}$ & 51 & 5 & P1 & 58,3 & 4,9 & 0,057 & 5,2 & $-13,9$ \\
\hline & & & & PPM & 52,8 & 8,2 & 0,074 & 4,0 & $-14,2$ \\
\hline \multirow[t]{2}{*}{2396} & $\mathrm{~F}$ & 18 & 5 & $\mathrm{P} 1$ & 63,3 & 5,4 & 0,048 & 4,0 & $-13,5$ \\
\hline & & & & PPMd & 31,7 & 6,1 & 0,047 & 3,5 & $-13,6$ \\
\hline \multirow[t]{12}{*}{2439} & $\mathrm{~F}$ & 54 & 5 & $\mathrm{C}$ & 63,5 & 5,2 & 0,046 & 4,0 & $-13,5$ \\
\hline & & & & Côte & 52,8 & 8,1 & 0,068 & 3,8 & $-14,2$ \\
\hline & & & Dents & Maximum & 69,8 & 6,1 & 0,061 & 6,1 & $-10,9$ \\
\hline & & & & Minimum & 42,3 & 2,7 & 0,024 & 3,6 & $-15,8$ \\
\hline & & & & Moyenne & 58,2 & 4,8 & 0,047 & 4,5 & $-13,9$ \\
\hline & & & & Ecart-type & 7,5 & 0,9 & 0,01 & 0,6 & 0,8 \\
\hline & & & & Médiane & 60,2 & 5,1 & 0,047 & 4,5 & $-13,8$ \\
\hline & & & Os & Maximum & 53,4 & 8,9 & 0,154 & 10,4 & $-11,3$ \\
\hline & & & & Minimum & 22,2 & 2,7 & 0,037 & 2,6 & $-14,9$ \\
\hline & & & & Moyenne & 36,9 & 6,5 & 0,072 & 5,1 & $-13,6$ \\
\hline & & & & Ecart-type & 9,0 & 1,5 & 0,03 & 1,8 & 0,8 \\
\hline & & & & Médiane & 37,2 & 6,4 & 0,067 & 4,8 & $-13,6$ \\
\hline
\end{tabular}

*les âges sont donnés en années, l'imprécision des âges individuels est de l'ordre de 4 ans, F : femme, H : homme ; Ind : indeterminé, HS : Hors secteur, $\mathrm{C}$ : canine ; $\mathrm{P} 1$ : première prémolaire ; $\mathrm{P} 2$ : deuxième prémolaire ; M1 : première molaire ; PPM : phalange proximale de la main, PMM : phalange médiale de la main ; PPP : phalange proximale du pied, MTT2, MTT3 ou MTT4 : métatarse 1, 2, 3 ou 4 ; d : droite ; $g$ : gauche. 
Tableau 3-Données élémentaires et isotopiques du corpus de faune - Elemental and isotopic data from the faunal corpus

\begin{tabular}{|c|c|c|c|c|c|c|c|c|}
\hline Lab Code & Espèce & Elément & $\begin{array}{c}\text { Rendement } \\
\left(\mathrm{mg.g}^{-1}\right)\end{array}$ & $\% \mathrm{C}$ & $\% \mathrm{~N}$ & $\mathbf{C} / \mathbf{N}$ & $\delta^{13} \mathrm{C}(\%)$ & $\delta^{15} \mathrm{~N}(\%)$ \\
\hline AIB.F09 & Bos taurus & Côte & 150,2 & 39,3 & 14,4 & 3,2 & $-22,1$ & 5,8 \\
\hline AIB.F22 & Bos taurus & Mandibule & 12,6 & 28,9 & 10,9 & 3,1 & $-21,9$ & 2,8 \\
\hline AIB.F26 & Bos taurus & Mandibule & 177,4 & 43,9 & 16,2 & 3,2 & $-22,5$ & 6,4 \\
\hline AIB.F30 & Bos taurus & PP & 31,4 & 41,8 & 15,5 & 3,1 & $-22,5$ & 6,8 \\
\hline AIB.F31 & Bos taurus & Vertèbre & 50,5 & 42,7 & 15,8 & 3,1 & $-22,3$ & 7,8 \\
\hline AIB.F32 & Bos taurus & Axis & 65,6 & 41,8 & 15,2 & 3,2 & $-21,6$ & 7,9 \\
\hline AIB.F33 & Bos taurus & Vertèbre & 31,1 & 41,3 & 15,0 & 3,2 & $-22,5$ & 7,5 \\
\hline AIB.F34 & Bos taurus & Vertèbre & 38,6 & 39,8 & 14,4 & 3,2 & $-22,7$ & 7,3 \\
\hline AIB.F35 & Bos taurus & Vertèbre & 61,1 & 44,6 & 16,6 & 3,1 & $-22,6$ & 8,2 \\
\hline DU.F06 & Bos taurus & Vertèbre & 34,5 & 37,3 & 13,9 & 3,1 & $-22,6$ & 6,4 \\
\hline \multirow[t]{6}{*}{ DU.F08 } & Bos taurus & Scapula & 42,7 & 40,6 & 14,9 & 3,2 & $-21,8$ & 4,2 \\
\hline & $\mathrm{n}=11$ & Maximum & 177,4 & 44,6 & 16,6 & 3,2 & $-21,6$ & 8,2 \\
\hline & & Minimum & 12,6 & 28,9 & 10,9 & 3,1 & $-22,7$ & 2,8 \\
\hline & & Moyenne & 63,2 & 40,2 & 14,8 & 3,2 & $-22,3$ & 6,5 \\
\hline & & Ecart-type & 52,2 & 4,3 & 1,5 & 0,0 & 0,4 & 1,7 \\
\hline & & Médiane & 42,7 & 41,3 & 15,0 & 3,2 & $-22,5$ & 6,8 \\
\hline AIB.F03 & Ovis aries & Fémur & 62,7 & 39,0 & 14,3 & 3,2 & $-22,4$ & 5,9 \\
\hline AIB.F04 & Ovis aries & MTT & 120,5 & 42,2 & 15,6 & 3,1 & $-21,5$ & 7,7 \\
\hline \multirow[t]{6}{*}{ AIB.F37 } & Ovis aries & MTC & 54,5 & 41,5 & 15,4 & 3,1 & $-21,5$ & 8,9 \\
\hline & $\mathrm{n}=3$ & Maximum & 120,5 & 42,2 & 15,6 & 3,2 & $-21,5$ & 8,9 \\
\hline & & Minimum & 54,5 & 39,0 & 14,3 & 3,1 & $-22,4$ & 5,9 \\
\hline & & Moyenne & 79,2 & 40,9 & 15,1 & 3,2 & $-21,8$ & 7,5 \\
\hline & & Ecart-type & 36,0 & 1,7 & 0,7 & 0,0 & 0,5 & 1,5 \\
\hline & & Médiane & 62,7 & 41,5 & 15,4 & 3,1 & $-21,5$ & 7,7 \\
\hline AIB.F108 & Equus caballus & Atlas & 35,6 & 42,4 & 15,6 & 3,2 & $-22,2$ & 5,0 \\
\hline \multirow[t]{6}{*}{ DU.F07 } & Equus caballus & Mandibule & 31,8 & 34,9 & 13,0 & 3,1 & $-21,3$ & 5,1 \\
\hline & $n=2$ & Maximum & 35,6 & 42,4 & 15,6 & 3,2 & $-21,3$ & 5,1 \\
\hline & & Minimum & 31,8 & 34,9 & 13,0 & 3,1 & $-22,2$ & 5,0 \\
\hline & & Moyenne & 33,7 & 38,6 & 14,3 & 3,1 & $-21,7$ & 5,0 \\
\hline & & Ecart-type & 2,7 & 5,3 & 1,8 & 0,0 & 0,6 & 0,1 \\
\hline & & Médiane & 33,7 & 38,6 & 14,3 & 3,1 & $-21,7$ & 5,0 \\
\hline AIB.F13 & Sus scrofa & MTC & 136,0 & 38,3 & 14,1 & 3,2 & $-21,5$ & 5,6 \\
\hline AIB.F12 & Sus scrofa & MTT & 162,8 & 41,3 & 15,2 & 3,2 & $-20,6$ & 6,0 \\
\hline AIB.F14 & Sus scrofa & MTT & 142,8 & 41,1 & 15,2 & 3,2 & $-21,4$ & 5,8 \\
\hline AIB.F15 & Sus scrofa & MTT & 134,2 & 41,7 & 15,2 & 3,2 & $-21,4$ & 5,9 \\
\hline AIB.F16 & Sus scrofa & Tibia & 116,8 & 39,6 & 14,5 & 3,2 & $-21,3$ & 5,6 \\
\hline AIB.F17 & Sus scrofa & Mandibule & 143,7 & 41,3 & 15,0 & 3,2 & $-21,5$ & 5,7 \\
\hline AIB.F19 & Sus scrofa & Mandibule & 154,1 & 40,6 & 14,9 & 3,2 & $-21,1$ & 6,0 \\
\hline AIB.F24 & Sus scrofa & Mandibule & 135,2 & 43,7 & 15,9 & 3,2 & $-21,3$ & 4,7 \\
\hline AIB.F25 & Sus scrofa & Os long & 107,7 & 45,1 & 16,4 & 3,2 & $-21,5$ & 5,6 \\
\hline \multirow[t]{6}{*}{ AIB.F27 } & Sus scrofa & MTT & 87,6 & 37,9 & 13,7 & 3,2 & $-21,2$ & 5,1 \\
\hline & $\mathrm{n}=10$ & Maximum & 162,8 & 45,1 & 16,4 & 3,2 & $-20,6$ & 6,0 \\
\hline & & Minimum & 87,6 & 37,9 & 13,7 & 3,2 & $-21,5$ & 4,7 \\
\hline & & Moyenne & 132,1 & 41,1 & 15,0 & 3,2 & $-21,3$ & 5,6 \\
\hline & & Ecart-type & 22,4 & 2,2 & 0,8 & 0,0 & 0,3 & 0,4 \\
\hline & & Médiane & 135,6 & 41,2 & 15,1 & 3,2 & $-21,4$ & 5,7 \\
\hline AIB.F36 & Gallus sp. & Vertèbre & 37,1 & 43,4 & 15,3 & 3,3 & $-20,7$ & 9,4 \\
\hline \multirow[t]{3}{*}{ DU.F01 } & Canis familiaris & Tibia & 174,4 & 39,9 & 14,5 & 3,2 & $-20,0$ & 10,0 \\
\hline & Faune totale & Maximum & 177,4 & 45,1 & 16,6 & 3,3 & $-20,0$ & 10,0 \\
\hline & $\mathrm{n}=28$ & Minimum & 12,6 & 28,9 & 10,9 & 3,1 & $-22,7$ & 2,8 \\
\hline
\end{tabular}




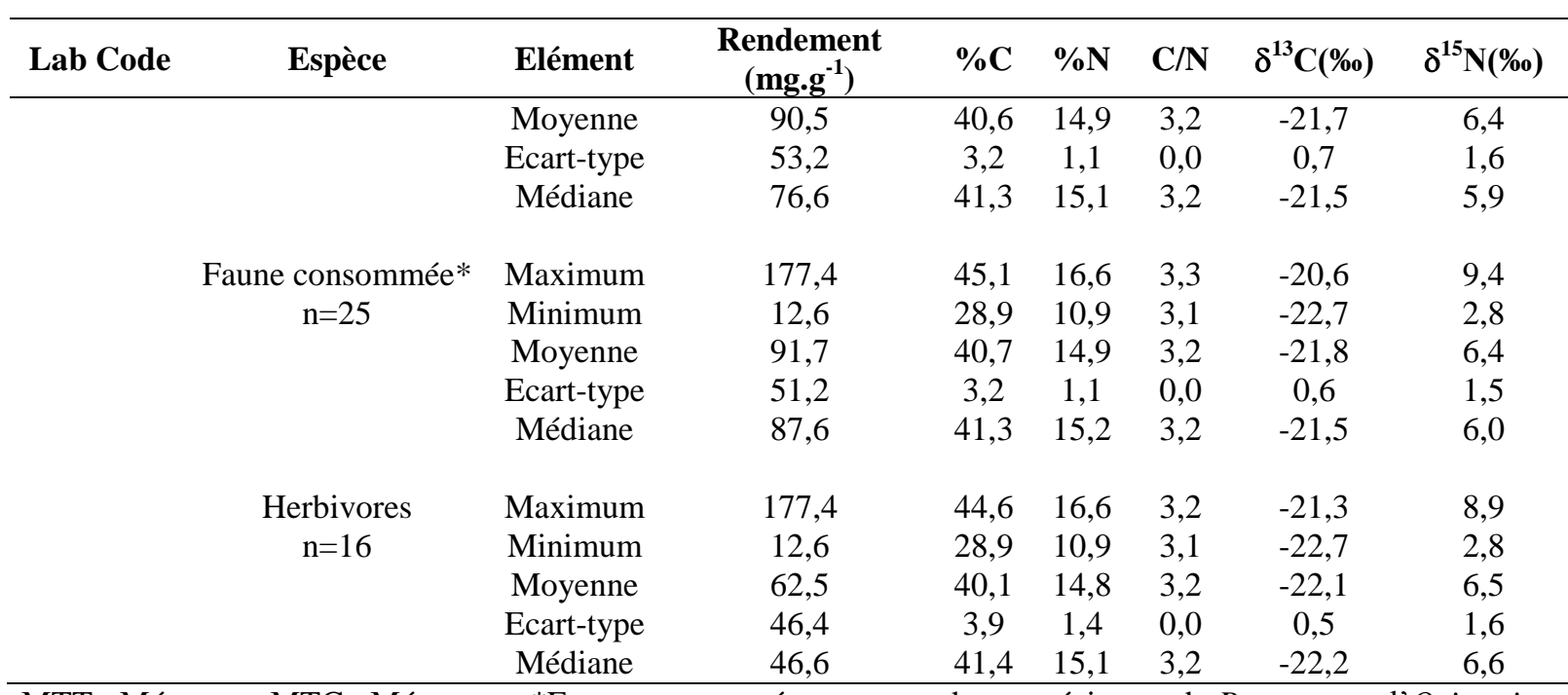

MTT : Métatarse, MTC : Métacarpe. *Faune consommée correspond aux spécimens de Bos taurus, d'Ovis aries, de Sus scrofa et de Gallus sp. 
Tableau 4-Résultats des tests de corrélation de rang de Spearman - Spearman correlation tests results

\begin{tabular}{|c|c|c|c|c|}
\hline & Facteur considéré & $\mathbf{S}$ & rho & p-value \\
\hline \multirow{4}{*}{$\delta^{13} C_{\text {faune }}$} & $\mathrm{Rdt}_{\mathrm{co}}$ & 1944,5 & 0,47 & 0,01 \\
\hline & $\% \mathrm{C}$ & 3902,6 & $-0,07$ & 0,73 \\
\hline & $\% \mathrm{~N}$ & 3974,1 & $-0,09$ & 0,66 \\
\hline & $\mathrm{C} / \mathrm{N}$ & 2196,2 & 0,40 & 0,04 \\
\hline \multirow{4}{*}{$\delta^{15} \mathbf{N}_{\text {faune }}$} & $\mathrm{Rdt}_{\mathrm{co}}$ & 3561,0 & 0,03 & 0,90 \\
\hline & $\% \mathrm{C}$ & 2349,5 & 0,36 & 0,06 \\
\hline & $\% \mathrm{~N}$ & 2490,8 & 0,32 & 0,10 \\
\hline & $\mathrm{C} / \mathrm{N}$ & 4125,6 & $-0,13$ & 0,51 \\
\hline \multirow{5}{*}{$\delta^{13} \mathrm{C}_{\mathrm{co} / \mathrm{os}}$} & $\mathrm{Rdt}_{\mathrm{co}}$ & 5592,8 & 0,07 & 0,72 \\
\hline & $\% \mathrm{C}$ & 5911,4 & 0,01 & 0,95 \\
\hline & $\% \mathrm{~N}$ & 5829,2 & 0,03 & 0,89 \\
\hline & $\mathrm{C} / \mathrm{N}$ & 6160,6 & $-0,03$ & 0,87 \\
\hline & Age & 4261,4 & 0,29 & 0,10 \\
\hline \multirow[t]{5}{*}{$\delta^{15} \mathbf{N}_{\text {os }}$} & $\mathrm{Rdt}_{\mathrm{co}}$ & 4725,9 & 0,21 & 0,24 \\
\hline & $\% \mathrm{C}$ & 4594,1 & 0,23 & 0,19 \\
\hline & $\% \mathrm{~N}$ & 4952,6 & 0,17 & 0,34 \\
\hline & $\mathrm{C} / \mathrm{N}$ & 3505,2 & 0,41 & 0,02 \\
\hline & Age & 7035,1 & $-0,18$ & 0,33 \\
\hline \multirow{5}{*}{$\delta^{13} \mathrm{C}_{\mathrm{ap} / \mathrm{os}}$} & $\mathrm{Rdt}_{\mathrm{ap}}$ & 7413,2 & $-0,24$ & 0,18 \\
\hline & $\mathrm{Pds}$ & 6155,0 & $-0,03$ & 0,87 \\
\hline & $\mathrm{Vol} \mathrm{CO}{ }_{2}$ & 3363,5 & 0,44 & 0,01 \\
\hline & $\% \mathrm{CO}_{3}$ & 3539,2 & 0,41 & 0,02 \\
\hline & Age & 6798,5 & $-0,14$ & 0,45 \\
\hline \multirow{5}{*}{$\delta^{13} \mathrm{C}_{\mathrm{co} / \mathrm{dt}}$} & $\mathrm{Rdt}_{\mathrm{co}}$ & 3703,5 & 0,38 & 0,03 \\
\hline & $\% \mathrm{C}$ & 4266,6 & 0,29 & 0,11 \\
\hline & $\% \mathrm{~N}$ & 4104,8 & 0,31 & 0,08 \\
\hline & $\mathrm{C} / \mathrm{N}$ & 7063,8 & $-0,18$ & 0,31 \\
\hline & Age & 4393,2 & 0,27 & 0,13 \\
\hline \multirow[t]{5}{*}{$\delta^{15} \mathbf{N}_{\mathrm{dt}}$} & $\mathrm{Rdt}_{\mathrm{co}}$ & 5457,9 & 0,09 & 0,63 \\
\hline & $\% \mathrm{C}$ & 7212,7 & $-0,21$ & 0,25 \\
\hline & $\% \mathrm{~N}$ & 7041,4 & $-0,18$ & 0,33 \\
\hline & $\mathrm{C} / \mathrm{N}$ & 4962,8 & 0,17 & 0,34 \\
\hline & Age & 7481,9 & $-0,25$ & 0,16 \\
\hline \multirow{5}{*}{$\delta^{13} \mathrm{C}_{\mathrm{ap} / \mathrm{dt}}$} & $\mathrm{Rdt}_{\mathrm{ap}}$ & 4726,8 & 0,21 & 0,24 \\
\hline & $\mathrm{Pds}$ & 5424,0 & 0,09 & 0,60 \\
\hline & $\mathrm{Vol} \mathrm{CO}$ & 5222,7 & 0,13 & 0,48 \\
\hline & $\% \mathrm{CO}_{3}$ & 5777,9 & 0,03 & 0,85 \\
\hline & Age & 5728,9 & 0,04 & 0,81 \\
\hline
\end{tabular}

Résultats significatifs en gras, corrélation par convention comme forte seulement si rho $>0,6$ 
Tableau 5-Résultats des tests approximatifs (Monte Carlo, 10000 réplicats) de Kruskal Wallis - Approximative Kruskal Wallis test results (Monte Carlo, 10000 replicats)

\begin{tabular}{lcc}
\hline \multicolumn{2}{l}{ Chi $^{\mathbf{2}}$} & p-value \\
\hline $\begin{array}{l}\text { Bos taurus }(\mathbf{n = 1 1}), \text { Equus caballus }(\mathbf{n}=\mathbf{2}), \\
\text { Ovis aries }(\mathbf{n = 3})\end{array}$ & \\
$\delta^{13} \mathrm{C}$ & 4,412 & 0,12 \\
$\delta^{15} \mathrm{~N}$ & 3,277 & 0,21 \\
Secteurs $\mathbf{1}(\mathbf{n = 1}), \mathbf{2}(\mathbf{n = 2}), \mathbf{3}(\mathbf{n = 3}), \mathbf{4}(\mathbf{n}=\mathbf{9})$, & & \\
$\mathbf{5}(\mathbf{n}=\mathbf{1 5}), \mathbf{6}(\mathbf{n = 2})$ et hors secteur $(\mathbf{n = 1})$ & & \\
$\delta^{13} \mathrm{C}_{\mathrm{co} / \mathrm{os}}$ & 11,528 & 0,19 \\
$\delta^{15} \mathrm{~N}_{\mathrm{os}}$ & 7,858 & 0,26 \\
$\delta^{13} \mathrm{C}_{\mathrm{ap} / \mathrm{os}}$ & 10,615 & 0,39 \\
$\delta^{13} \mathrm{C}_{\mathrm{co} / \mathrm{dt}}$ & 6,632 & 0,35 \\
$\delta^{15} \mathrm{~N}_{\mathrm{dt}}$ & 7,383 & 0,61 \\
$\delta^{13} \mathrm{C}_{\mathrm{ap} / \mathrm{dt}}$ & 2,892 & 0,88 \\
\hline
\end{tabular}

Tableau 6-Résultats des tests exacts de Wilcoxon bivariés - Wilcoxon exact unpaired tests results

\begin{tabular}{lrr}
\hline & \multicolumn{1}{c}{$\mathbf{Z}$} & p-value \\
\hline Herbivores $(\mathbf{n}=\mathbf{1 6})$, Sus scrofa $(\mathbf{n = 1 0})$ & & \\
$\delta^{13} \mathrm{C}$ (différence $\left.>0\right)$ & 3,8224 & $\mathbf{9 . 4 e - 0 6}$ \\
$\delta^{15} \mathrm{~N}$ (différence <0) & $-1,9768$ & $\mathbf{0 . 0 2}$ \\
Hommes $(\mathbf{n = 1 5})$, femmes $(\mathbf{n = 1 7 )}$ & & \\
$\delta^{13} \mathrm{C}_{\mathrm{co} / \mathrm{os}}$ & 0,5289 & 0,61 \\
$\delta^{15} \mathrm{~N}_{\mathrm{os}}$ & $-0,3777$ & 0,72 \\
$\delta^{13} \mathrm{C}_{\mathrm{ap} / \mathrm{os}}$ & 1,6427 & 0,11 \\
$\delta^{13} \mathrm{C}_{\mathrm{co} / \mathrm{dt}}$ & 0,8312 & 0,42 \\
$\delta^{15} \mathrm{~N}_{\mathrm{dt}}$ & $-0,2455$ & 0,82 \\
$\delta^{13} \mathrm{C}_{\mathrm{ap} / \mathrm{dt}}$ & 0,1511 & 0,89 \\
\hline
\end{tabular}

Tableau 7-Résultats des tests exacts de Wilcoxon appariés- Wilcoxon exact paired tests results

\begin{tabular}{ccc}
\hline & $\mathbf{Z}$ & $\mathbf{p}$ value \\
\hline Dents $(\mathbf{n}=\mathbf{3 3})$, os $(\mathbf{n = 3 3})$ & & \\
$\delta^{13} \mathrm{C}_{\mathrm{co}}$ & $-1,644$ & 0,10 \\
$\delta^{15} \mathrm{~N}$ & 3,5827 & $\mathbf{0 , 0 0}$ \\
$\delta^{13} \mathrm{C}_{\mathrm{ap}}$ & 0,83095 & 0,41 \\
\hline
\end{tabular}


Tableau 8-Statistiques récapitulatives des valeurs isotopiques des groupes humains Statistics of isotope values acccording to human groups

\begin{tabular}{|c|c|c|c|c|c|c|c|}
\hline & & $\delta^{13} \mathrm{C}_{\mathrm{co} / \mathrm{dt} t}(\%)$ & $\delta^{15} N_{d t}(\%)$ & $\delta^{13} \mathrm{C}_{\mathrm{ap} / \mathrm{dt}}(\% \mathrm{o})$ & $\delta^{13} \mathrm{C}_{\mathrm{co} / \mathrm{os}}(\%)$ & $\delta^{15} \mathbf{N}_{o s}(\%)$ & $\delta^{13} C_{a p / o s}(\%)$ \\
\hline \multicolumn{8}{|l|}{ Sexe } \\
\hline \multirow{5}{*}{$\begin{array}{c}\text { Femmes } \\
n=17\end{array}$} & Maximum & $-15,3$ & 12,3 & $-11,3$ & $-15,1$ & 10,9 & $-10,9$ \\
\hline & Minimum & $-19,8$ & 7,4 & $-14,6$ & $-19,9$ & 7,5 & $-15,2$ \\
\hline & Moyenne & $-19,3$ & 9,3 & $-13,6$ & $-19,3$ & 9,7 & $-13,7$ \\
\hline & Ecart-type & 1,1 & 1,1 & 0,9 & 1,1 & 0,9 & 0,9 \\
\hline & Médiane & $-19,5$ & 9,1 & $-13,6$ & $-19,5$ & 9,7 & $-13,8$ \\
\hline \multirow{5}{*}{$\begin{array}{c}\text { Hommes } \\
n=15\end{array}$} & Maximum & $-19,2$ & 12,3 & $-12,7$ & $-18,9$ & 12,9 & $-13,3$ \\
\hline & Minimum & $-20,4$ & 8,2 & $-14,9$ & $-20,2$ & 8,3 & $-15,8$ \\
\hline & Moyenne & $-19,6$ & 9,4 & $-13,7$ & $-19,6$ & 10,0 & $-14,2$ \\
\hline & Ecart-type & 0,3 & 1,0 & 0,7 & 0,3 & 1,0 & 0,7 \\
\hline & Médiane & $-19,7$ & 9,4 & $-13,8$ & $-19,5$ & 9,9 & $-14,0$ \\
\hline Ind. & $\mathrm{n}=1$ & $-19,5$ & 9,7 & $-13,0$ & $-19,1$ & 10,5 & $-13,4$ \\
\hline \multicolumn{8}{|c|}{ Secteur d'inhumation } \\
\hline Secteur 1 & $\mathrm{n}=1$ & $-19,6$ & 9,9 & $-14,4$ & $-19,4$ & 9,9 & $-13,3$ \\
\hline \multirow{5}{*}{$\begin{array}{c}\text { Secteur } 2 \\
n=2\end{array}$} & Maximum & $-19,3$ & 10,5 & $-14,2$ & $-19,3$ & 10,7 & $-13,8$ \\
\hline & Minimum & $-19,4$ & 9,4 & $-14,5$ & $-19,4$ & 9,4 & $-13,9$ \\
\hline & Moyenne & $-19,3$ & 9,9 & $-14,4$ & $-19,4$ & 10,1 & $-13,9$ \\
\hline & Ecart-type & 0,0 & 0,8 & 0,2 & 0,1 & 0,9 & 0,0 \\
\hline & Médiane & $-19,3$ & 9,9 & $-14,4$ & $-19,4$ & 10,1 & $-13,9$ \\
\hline \multirow{5}{*}{$\begin{array}{c}\text { Secteur } 3 \\
n=3\end{array}$} & Maximum & $-19,6$ & 9,3 & $-12,7$ & $-19,3$ & 9,5 & $-13,8$ \\
\hline & Minimum & $-19,8$ & 8,6 & $-13,4$ & $-19,7$ & 8,6 & $-14,3$ \\
\hline & Moyenne & $-19,8$ & 8,8 & $-13,2$ & $-19,5$ & 8,9 & $-14,0$ \\
\hline & Ecart-type & 0,1 & 0,4 & 0,4 & 0,2 & 0,5 & 0,2 \\
\hline & Médiane & $-19,8$ & 8,6 & $-13,3$ & $-19,5$ & 8,7 & $-14,0$ \\
\hline \multirow{5}{*}{$\begin{array}{c}\text { Secteur } 4 \\
n=9\end{array}$} & Maximum & $-18,7$ & 12,3 & $-12,7$ & $-19,1$ & 12,9 & $-12,7$ \\
\hline & Minimum & $-19,8$ & 8,6 & $-13,4$ & $-19,7$ & 8,6 & $-14,3$ \\
\hline & Moyenne & $-19,8$ & 8,8 & $-13,2$ & $-19,5$ & 8,9 & $-14,0$ \\
\hline & Ecart-type & 0,1 & 0,4 & 0,4 & 0,2 & 0,5 & 0,2 \\
\hline & Médiane & $-19,8$ & 8,6 & $-13,3$ & $-19,5$ & 8,7 & $-14,0$ \\
\hline \multirow{5}{*}{$\begin{array}{c}\text { Secteur } 5 \\
n=15\end{array}$} & Maximum & $-15,3$ & 12,3 & $-11,3$ & $-15,1$ & 10,9 & $-10,9$ \\
\hline & Minimum & $-20,4$ & 8,2 & $-14,9$ & $-20,2$ & 8,5 & $-15,4$ \\
\hline & Moyenne & $-19,3$ & 9,2 & $-13,6$ & $-19,2$ & 9,9 & $-13,9$ \\
\hline & Ecart-type & 1,1 & 1,0 & 0,8 & 1,2 & 0,7 & 1,0 \\
\hline & Médiane & $-19,5$ & 9,1 & $-13,6$ & $-19,5$ & 10,1 & $-13,9$ \\
\hline \multirow{5}{*}{$\begin{array}{c}\text { Secteur } 6 \\
n=2\end{array}$} & Maximum & $-19,5$ & 10,1 & $-12,7$ & $-19,8$ & 10,2 & $-13,8$ \\
\hline & Minimum & $-19,6$ & 9,7 & $-13,3$ & $-19,8$ & 9,6 & $-14,0$ \\
\hline & Moyenne & $-19,6$ & 9,9 & $-13,0$ & $-19,8$ & 9,9 & $-13,9$ \\
\hline & Ecart-type & 0,1 & 0,3 & 0,4 & 0,0 & 0,4 & 0,1 \\
\hline & Médiane & $-19,6$ & 9,9 & $-13,0$ & $-19,8$ & 9,9 & $-13,9$ \\
\hline Hors secteur & $\mathrm{n}=1$ & $-19,6$ & 7,4 & $-14,6$ & $-19,5$ & 7,5 & $-13,8$ \\
\hline
\end{tabular}




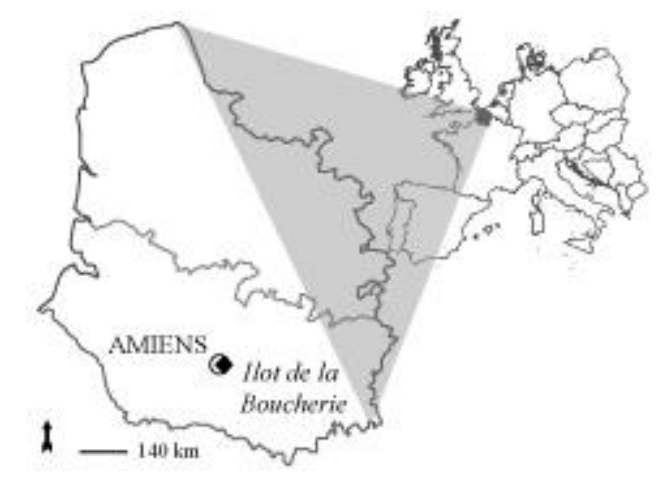

Figure 1-Situation géographique du site archéologique de l'Ilot de la Boucherie, Amiens, Somme, France - Geographic context of the archaeological site of Ilot de la Boucherie, Amiens, Somme, France

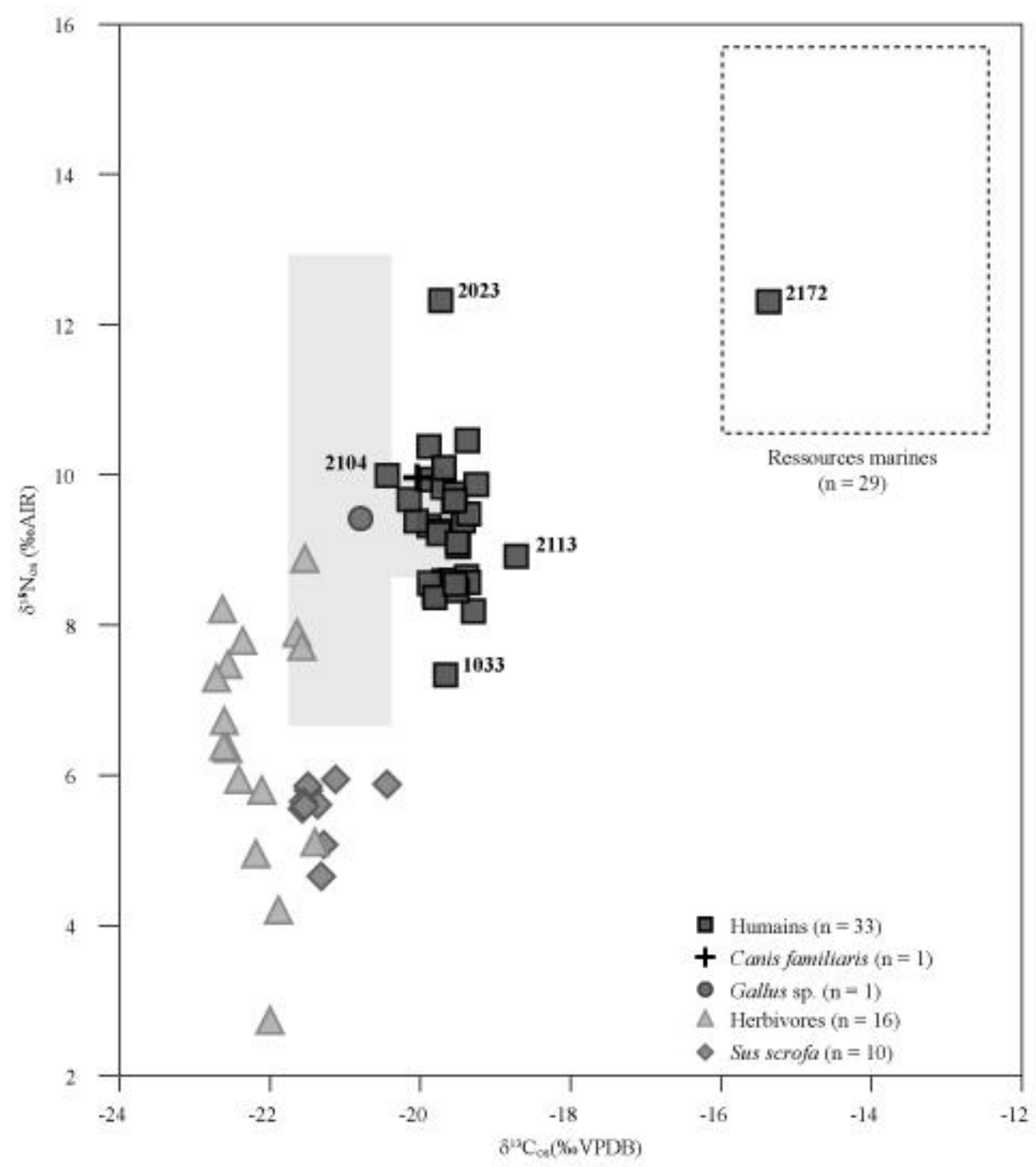

Figure 2-Valeurs isotopiques $\left(\delta^{13} \mathrm{C}_{\mathrm{co}} / \delta^{15} \mathrm{~N}\right.$, os ) des sujets d'Amiens, du corpus de faune et des ressources marines publiées pour la Manche [65,66]-Isotope values $\left(\delta^{13} \mathrm{C}_{\mathrm{co}} / \delta^{15} \mathrm{~N}\right.$, bone) from 
the subjects of Amiens, the faunal corpus and the published marine ressources of the Channel Sea $[65,66]$

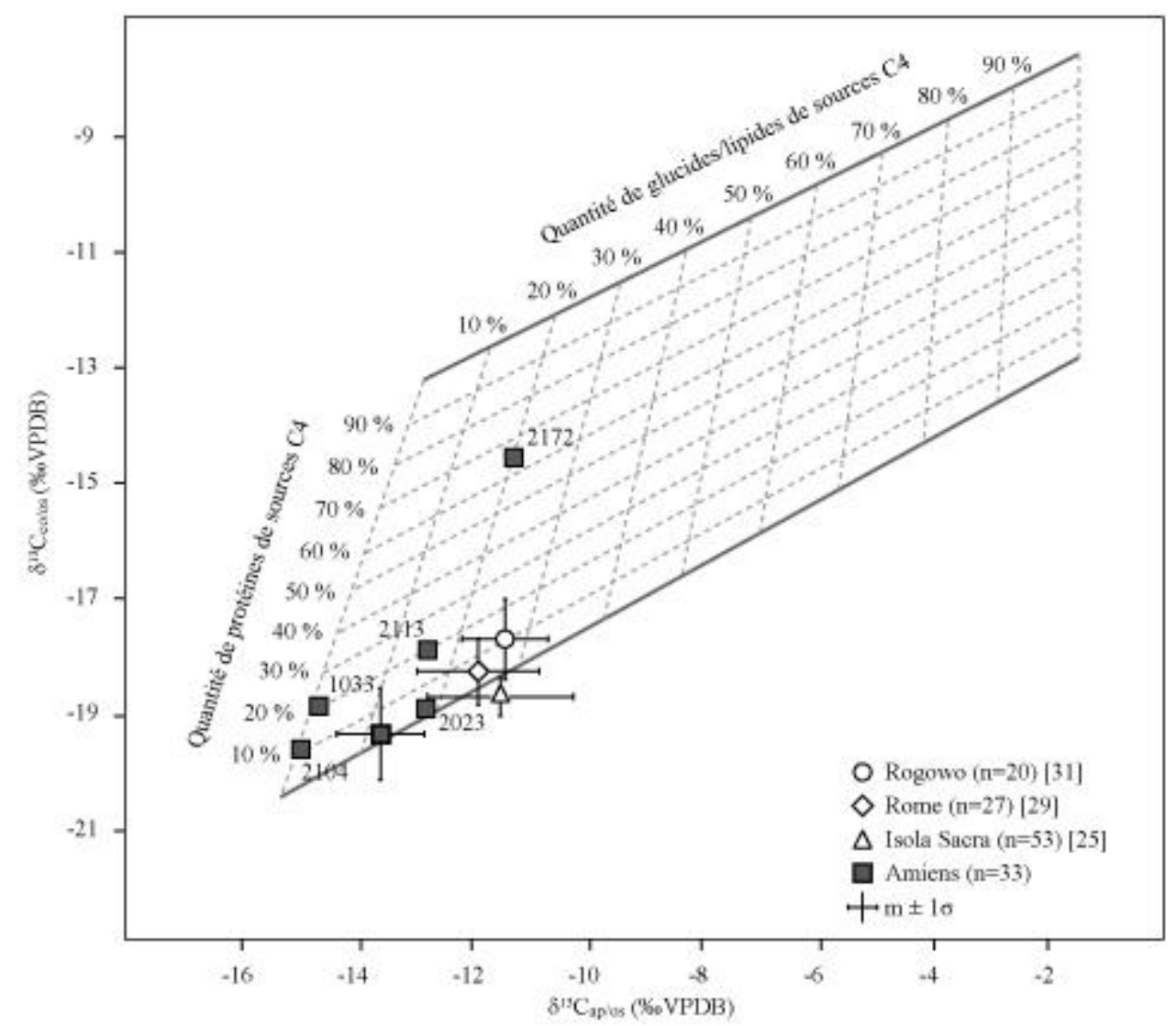

Figure 3-Valeurs isotopiques du carbone (os) selon le modèle mono-isotopique $[43,44,57]$ Carbon isotope values (bone) according to the simple carbon isotope model $[43,44,57]$ 


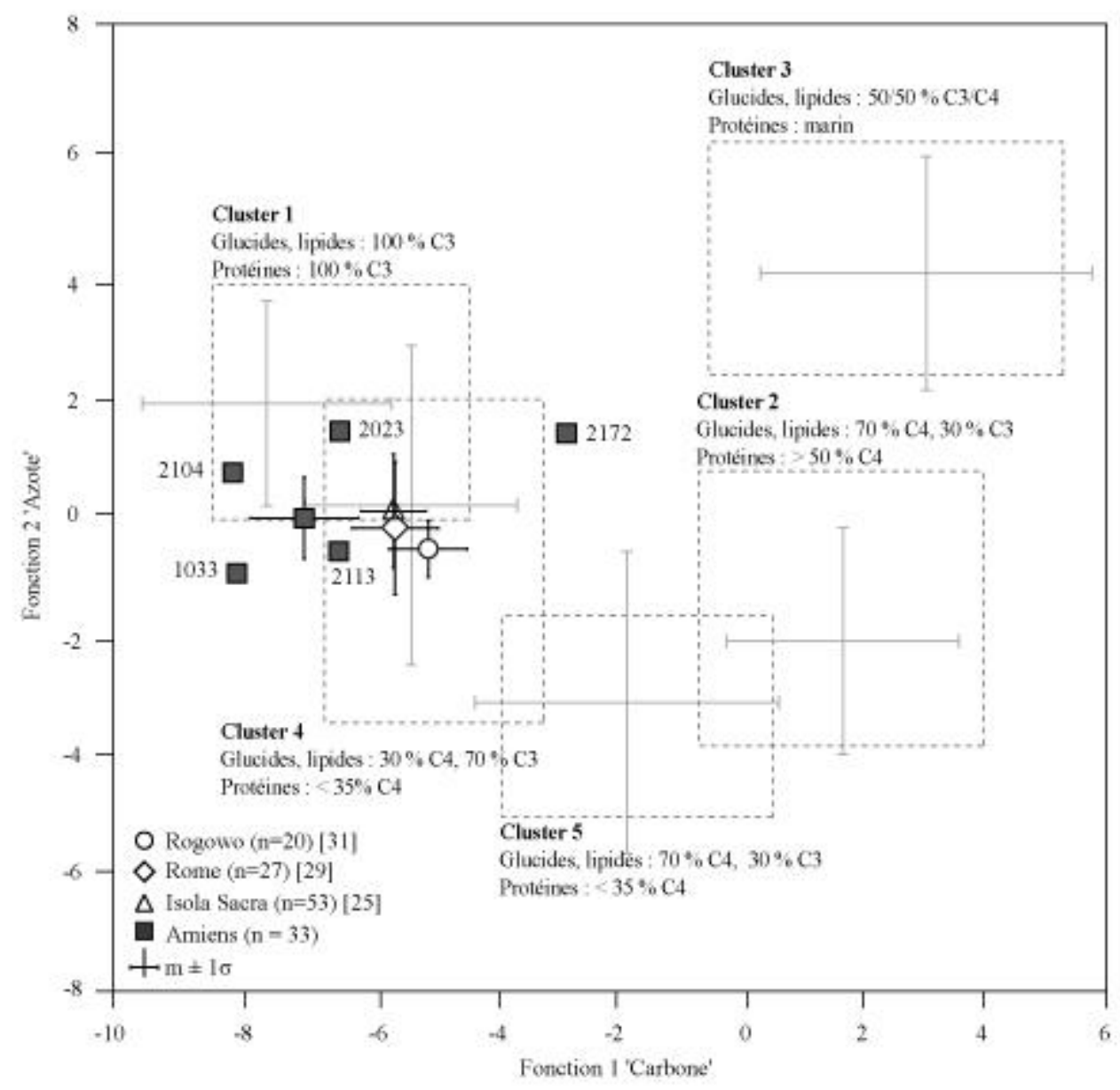

Figure 4-Valeurs isotopiques $\left(\delta^{13} \mathrm{C}_{\mathrm{ap}}, \delta^{13} \mathrm{C}_{\mathrm{co}}\right.$ et $\delta^{15} \mathrm{~N}$, os) selon le modèle multi -isotopique des fonctions discriminantes [56] - Isotope values $\left(\delta^{13} \mathrm{C}_{\mathrm{ap}}, \delta^{13} \mathrm{C}_{\mathrm{co}}\right.$ and $\delta^{15} \mathrm{~N}$, bone $)$ according to the multi -isotopic model of discriminant functions [56] 


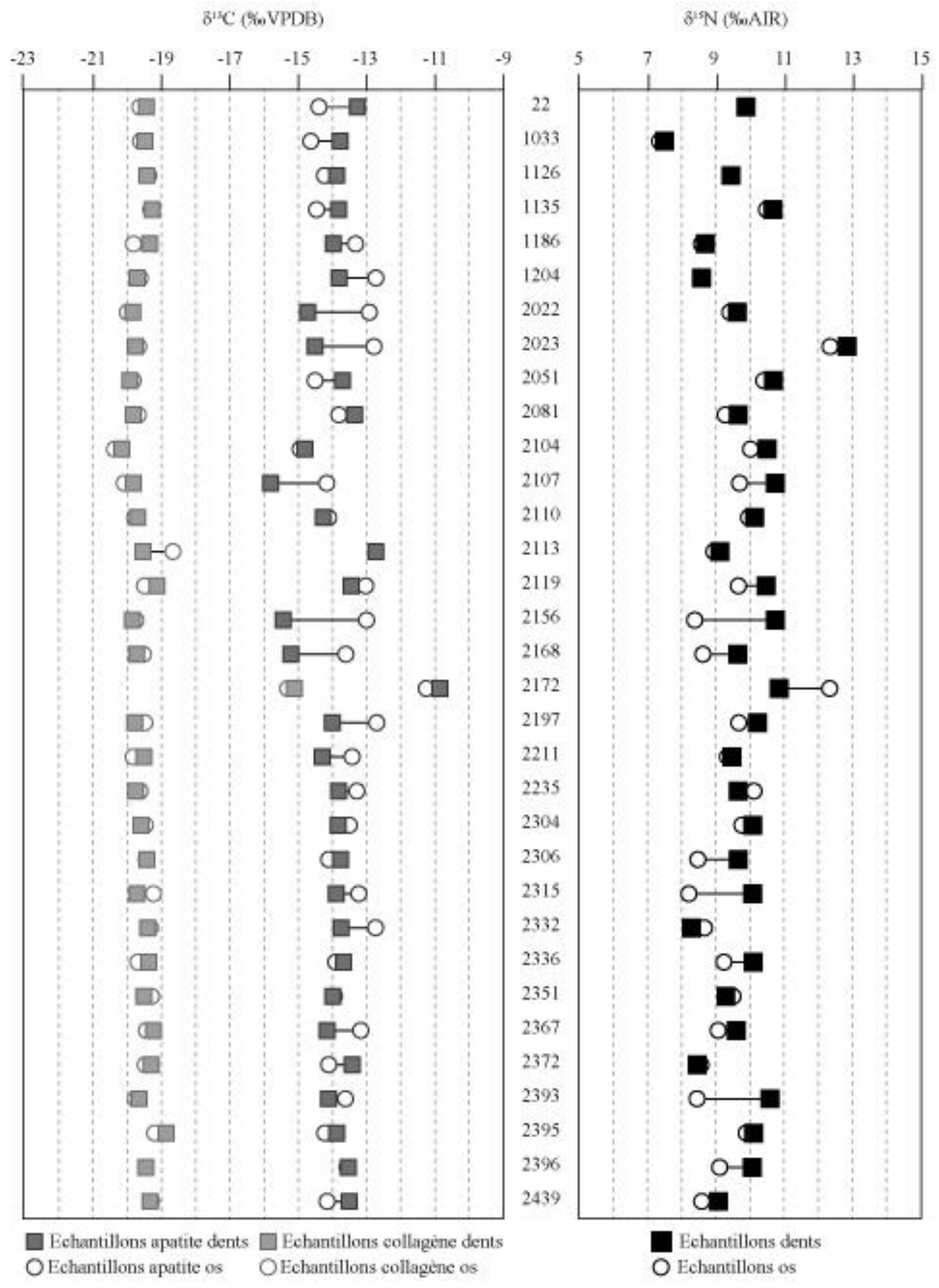

Figure 5-Comparaison des valeurs isotopiques $\left(\delta^{13} \mathrm{C}_{\mathrm{ap}}, \delta^{13} \mathrm{C}_{\mathrm{co}}\right.$ et $\left.\delta^{15} \mathrm{~N}\right)$ des tissus osseux et dentaires pour les 33 individus humains étudiés-Comparison of isotope values $\left(\delta^{13} \mathrm{C}_{\mathrm{ap}}, \delta^{13} \mathrm{C}_{\mathrm{co}}\right.$ and $\delta^{15} \mathrm{~N}$ ) from bone and dental tissus of the 33 human specimen studied 